\title{
R Coronae Borealis stars in the Galactic bulge discovered by EROS-2 ${ }^{\star}$
}

P. Tisserand ${ }^{1,2}$, J. B. Marquette ${ }^{3}$, P. R. Wood ${ }^{1}$, É. Lesquoy ${ }^{2,3}$, J. P. Beaulieu ${ }^{3}$, A. Milsztajn ${ }^{2, \star \star}$, C. Hamadache ${ }^{2}$, C. Afonso ${ }^{2, \star \star \star}$, J. N. Albert ${ }^{4}, J_{\text {. Andersen }}^{5}$, R. Ansari ${ }^{4}$, É. Aubourg ${ }^{2}$, P. Bareyre $^{2}$, X. Charlot $^{2}$, C. Coutures ${ }^{2,3}$, R. Ferlet ${ }^{3}$, P. Fouqué ${ }^{6}$, J. F. Glicenstein ${ }^{2}$, B. Goldmann, ${ }^{2}$, A. Gould ${ }^{8}$, M. Gros ${ }^{2}$, J. Haissinski ${ }^{4}$, J. de $\mathrm{Kat}^{2}$, L. Le Guillou ${ }^{2, \ddagger}$, C. Loup ${ }^{3, \S}$, C. Magneville ${ }^{2}$, É. Maurice ${ }^{9}$, A. Maury ${ }^{7, \mathbb{I}}$, M. Moniez $^{4}$, N. Palanque-Delabrouille ${ }^{2}$, O. Perdereau ${ }^{4}$, Y. Rahal ${ }^{4}$, J. Rich ${ }^{2}$, M. Spiro ${ }^{2}$, A. Vidal-Madjar ${ }^{3}$, and S. Zylberajch ${ }^{2}$

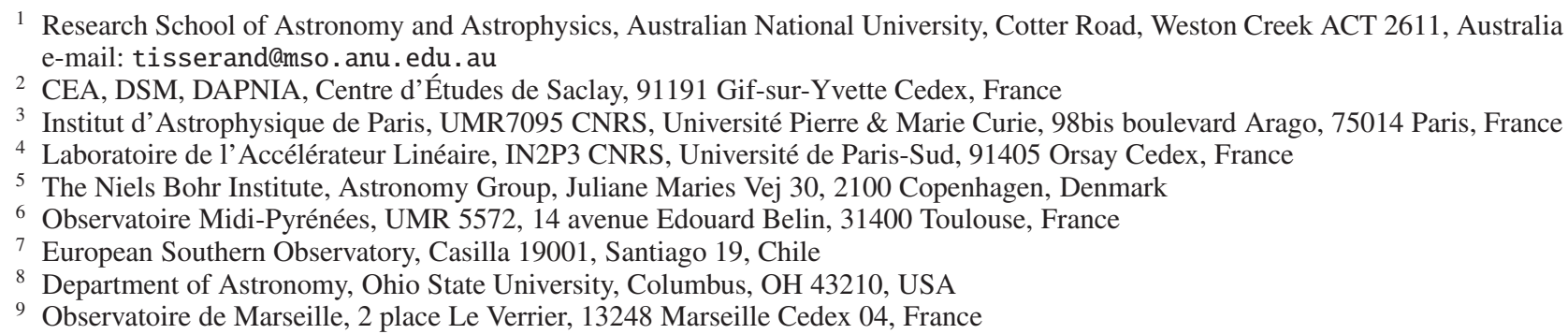

Received 9 October 2007 / Accepted 11 January 2008

\section{ABSTRACT}

Context. Rare types of variable stars may provide unique insight into short-lived stages of stellar evolution. The systematic monitoring of millions of stars and advanced light curve analysis techniques of microlensing surveys make them ideal for discovering such rare variable stars. One example is the R Coronae Borealis $(\mathrm{RCB})$ stars, a rare type of evolved carbon-rich supergiant.

Aims. We have conducted a systematic search of the EROS-2 database for the Galactic catalogue Bulge and spiral arms to find Galactic RCB stars.

Methods. The light curves of $\sim 100$ million stars, monitored for 6.7 years (from July 1996 to February 2003), have been analysed to search for the main signature of RCB stars, large and rapid drops in luminosity. Follow-up spectroscopy has been used to confirm the photometric candidates.

Results. We have discovered 14 new RCB stars, all in the direction of the Galactic Bulge, bringing the total number of confirmed Galactic RCB stars to about 51.

Conclusions. After reddening correction, the colours and absolute magnitudes of at least 9 of the stars are similar to those of Magellanic RCB stars. This suggests that these stars are in fact located in the Galactic Bulge, making them the first RCB stars discovered in the Bulge. The localisation of the 5 remaining RCBs is more uncertain: 4 are either located behind the Bulge at an estimated maximum distance of $14 \mathrm{kpc}$ or have an unusual thick circumstellar shell; the other is a DY Per RCB which may be located in the Bulge, even if it is fainter than the known Magellanic DY Per. From the small scale height found using the 9 new Bulge RCBs, $61<h_{\mathrm{Bulge}}^{\mathrm{RCB}}<246 \mathrm{pc}(95 \%$ C.L.), we conclude that the RCB stars follow a disk-like distribution inside the Bulge.

Key words. stars: carbon - stars: AGB and post-AGB - supergiants - Galaxy: bulge

* Based on observations made with the CNRS/INSU MARLY telescope at the European Southern Observatory, La Silla, Chile.

$\star \star$ Deceased.

$\star \star \star$ Now at Max-Planck-Institut für Astronomie, Koenigstuhl 17,

69117 Heidelberg, Germany.

Now at Max-Planck-Institut für Astronomie, Koenigstuhl 17, 69117 Heidelberg, Germany.

\$ Now at LPNHE, IN2P3 CNRS and Universités Paris 6 and Paris 7 , 4 place Jussieu, 75252 Paris Cedex 05, France.

$\S$ Now at Observatoire Astronomique de Strasbourg, UMR 7550,

11 rue de l'Université, 67000 Strasbourg, France.

II Now at San Pedro de Atacama Celestial Exploration, Casilla 21,

San Pedro de Atacama, Chile.

\section{Introduction}

The R Coronae Borealis (RCB) stars constitute a rare type of hydrogen deficient, carbon-rich supergiant star. Only 38 examples are currently known in the Galaxy (Clayton 1996; Zaniewski et al. 2005), 17 in the Large Magellanic Cloud (Alcock et al. 2001) and 6 in the Small Magellanic Cloud (Tisserand et al. 2004; Kraemer et al. 2005). This rarity presumably indicates a brief phase of stellar evolution. A detailed review of their characteristics has been written by Clayton (1996). RCBs exhibit spectacular, unpredictable, rapid declines in brightness (up to 9 mag in optical wavelengths over a few days or weeks) thought to be due to the photosphere being obscured by newly formed dust clouds along the line of sight. As the dust clouds disperse, the original brightness gradually recovers over months. Several of these clouds have been directly observed in various directions 
around the RCB star RY Sgr, see de Laverny \& Mékarnia (2004); Leão et al. (2007).

Up to now, there has been no reliable distance estimate to any Galactic RCB star, so their absolute magnitudes is only known from the RCBs discovered in the Magellanic Clouds (MC). A direct relation between effective temperature and absolute magnitude was observed by Alcock et al. (2001). Hipparcos gave lower limits to the distances to several of the brightest Galactic RCB stars (Trimble \& Kundu 1997; Cottrell \& Lawson 1998). Such limits imply that the RCB stars studied must be brighter than $M_{V}=-3$.

The true spatial distribution of RCB stars is still uncertain. This is due to the small number of known RCB stars, which is also biased toward higher Galactic latitudes as reddened stars in the Galactic plane can be missed in magnitude limited surveys (Lawson et al. 1990; Lawson \& Cottrell 1990). Different views are found in the literature. Iben \& Tutukov (1985) reported a scale height of $\sim 400$ pc for known RCB stars assuming $M_{\mathrm{Bol}}=-5$. Thus, RCB stars may be part of an old disk-like distribution. This idea is also supported by Zaniewski et al. (2005), who mention a possible thick-disk distribution. However, Cottrell \& Lawson (1998) noted that the Hipparcos velocity dispersion of RCB stars is similar to those of other cool hydrogen-deficient carbon stars and extreme helium (EHe) stars, suggesting that RCB stars might have a Bulge-like distribution.

Two major evolutionary scenarios are suggested to explain their origin: the double degenerate scenario (DD) and the final helium shell flash (FF) scenario (Iben et al. 1996; Renzini 1990). The DD model involves the merger of a CO- and a He-white dwarf and was recently strongly supported by the observations of ${ }^{18} \mathrm{O}$ over-abundance in seven H-deficient carbon and RCB stars, that is not expected in the FF model (Clayton et al. 2007). The birthrates of RCB stars would then help us to better understand the rates of mergers for objects that could be Supernovae type Ia progenitors (Webbink 1984; Belczynski et al. 2005). The FF model involves the expansion of a star, on the verge of becoming a white dwarf, to a supergiant size. This outburst phenomenon has been observed in three stars (Sakurai's object, V605 Aql and FG Sge), that transformed themselves into cool giant stars with spectral properties similar to those of RCB stars (Clayton \& De Marco 1997; Asplund et al. 1999; Gonzalez et al. 1998). However, Clayton et al. (2006) noted that differences between FF star and RCB star light curve variations and abundance patterns (such as ${ }^{12} \mathrm{C} /{ }^{13} \mathrm{C}$ ratio) indicate that the former are unlikely to be the evolutionary precursors of the majority of the latter.

One way to make progress in the comprehension of these stars is to enlarge the small sample of known objects. Microlensing surveys (OGLE, MACHO, MOA, EROS and Supermacho) are ideal for increasing the sample because they monitor millions of stars and thus could observe the characteristic RCB signature of rapid fading. The EROS-2 (Expérience de Recherche d'Objets Sombres) experiment is currently the survey that has monitored the largest sky area $\left(\sim 106 \mathrm{deg}^{2}\right.$ in our Galaxy) during a period of $\sim 6.7$ years. EROS can thus be used to significantly increase the number of known Galactic RCBs.

This article reports a sample of 14 new RCB stars. The photometric and spectroscopic data used are presented in Sect. 2. A discussion of the previously known RCB stars in and near the area monitored by EROS-2 is given in Sect. 3. Following the discovery of the first RCB stars in the SMC (Tisserand et al. 2004), we tried to increase our detection efficiency to prevent a selection effect due to reddening. The modified detection techniques and the spectroscopic observations are described in Sect. 4. The general characteristics of the new RCBs discovered are discussed in Sects. 5 and 6.

\section{Observational data}

The EROS-2 project used the 1-m MARLY telescope at ESO La Silla Observatory, Chile. The primary purpose of the project was to search for microlensing events (Paczynski 1986) due to baryonic dark matter in the halo (Tisserand et al. 2007) or to ordinary stars in the Galactic plane (Hamadache et al. 2006; Derue et al. 2001). The observations were performed between July 1996 and February 2003 with two wide field cameras $\left(0.69^{\circ}\right.$ in right ascension $\times 1.38^{\circ}$ in declination, thus a sky area of $\sim 0.95 \mathrm{deg}^{2}$ ) behind a dichroic cube splitting the light beam into two broad passbands. The so-called "blue" channel (420-720 nm, hereafter magnitudes $B_{\mathrm{E}}$ ) overlapped the $V$ and $R$ standard bands while the "red" one (620-920 nm, hereafter magnitudes $R_{\mathrm{E}}$ ) roughly matched the mean wavelength of the Cousins $I$ band. Each camera was a mosaic of eight $2048 \times 2048$ CCDs with a pixel size of $0.6^{\prime \prime}$ on the sky.

The photometric calibration was obtained directly for $20 \%$ of our fields by matching our star catalogues with those of the OGLE-II collaboration (Udalski et al. 2002). To a precision of $0.1 \mathrm{mag}$, we found the following transformations with the standard $V$ Johnson and $I$ Cousins broadband:

$R_{\mathrm{E}}=I, \quad B_{\mathrm{E}}=V-0.4(V-I)$.

The calibration is sufficiently uniform that it can be extended with confidence to the remaining fields. We also note that the OGLE calibration used a colour range of observed standard stars (Landolt system, Landolt 1992) limited to $V-I<2$ mag. For redder stars, they stressed that their transformations are extrapolations with a systematic error that may reach 0.25 mag at the very red edge $(V-I>4 \mathrm{mag})$. This is of particular interest for our study.

Five distinct sky areas have been monitored in our galaxy. The main sky area corresponds to 83 fields centred on the Galactic Centre (60 fields in the south Galactic side $(b<0)$ and 23 in the north one). These bulge fields are designated "cgxxxqq" where "xxx" is the number of the field and "qq" is the quadrant of a CCD. The remaining four zones are distributed in crowded regions along the spiral arms. They cover a surface of $\sim 27.5 \mathrm{deg}^{2}$ with 29 fields and are listed individually in Table 2 . The photometry of individual images and the reconstruction of the light curves were processed using the Peida package which has been specifically developed for the EROS experiment (Ansari 1996). A detailed discussion of the photometric accuracy can be found in Hamadache et al. (2006) for the Bulge fields and in Rahal (2003) for the spiral arms ones. The photometric precision for Bulge clump-giant stars is better than $2 \%$ for $R_{\mathrm{E}}<17$ and about $4 \%$ for $R_{\mathrm{E}} \sim 18$.

The template images are formed with 15 good seeing coadded images. They are used to detect sources and then form the initial object catalogue. With an exposure time of $120 \mathrm{~s}$ (except $\theta$ Muscae, $180 \mathrm{~s}$ ), our detection limit reaches about the $21 \mathrm{st}$ magnitude while stars brighter than the $\sim 11$ th magnitude are saturated. An average of one point every four nights was taken for each field during the Galactic Bulge observing season although the sampling frequency occasionally reached more than one point per night at mid-season. Stars were imaged on average at 800, 550 and 350 epochs for the cg, tm-gn and bs-gs fields respectively. Some low priority cg fields (18 out of the 83), less frequently observed (from $\sim 80$ to $\sim 200$ epochs), have also been considered in this analysis. 
Table 1. Known Galactic RCB stars located in the EROS-2 fields.

\begin{tabular}{llllc}
\hline \hline Star name & Coordinates $\left(\mathrm{J}_{2000}\right)$ & True MACHO Id & $\begin{array}{l}\text { Situation on EROS-2 } \\
\text { reference image }\end{array}$ & $\begin{array}{c}\text { Large variations } \\
\text { seen by EROS-2? }\end{array}$ \\
\hline V1783 Sgr & $18: 04: 49.74-32: 43: 13.6$ & & saturated, cg0842m & no \\
V739 Sgr & $18: 13: 10.54-30: 16: 14.7$ & MACHO-123.24377.7 & saturated, cg6245k & yes \\
V3795 Sgr & $18: 13: 23.58-25: 46: 40.8$ & MACHO-161.24445.6 & saturated, cg62731 & no \\
VZ Sgr & $18: 15: 08.58-29: 42: 29.4$ & MACHO-117.25166.4693 & saturated, cg0290n & yes \\
MACHO-135.27132.51 & $18: 19: 33.87-28: 35: 57.8$ & & too faint, cg0353k & yes \\
\hline
\end{tabular}

Table 2. Galactic sky areas monitored by EROS-2.

\begin{tabular}{lllll}
\hline \hline Sky area & $\begin{array}{l}\text { EROS2 } \\
\text { identifier }\end{array}$ & $\begin{array}{l}\text { Number } \\
\text { of fields }\end{array}$ & $\langle l\rangle$ & $\langle b\rangle$ \\
\hline Galactic Centre & cgxxx & 83 & 1.5 & -3.0 \\
$\gamma$ Scuti & gsxxx & 5 & 18.71 & 2.66 \\
$\beta$ Scuti & bsxxx & 6 & 27.75 & 2.64 \\
$\gamma$ Normae & gnxxx & 12 & 331.38 & 2.85 \\
$\theta$ Muscae & tmxxx & 6 & 306.85 & 1.78 \\
\hline
\end{tabular}

Spectroscopy of RCB candidates was performed with the Dual-Beam Spectrograph (DBS) (Rodgers et al. 1988) attached to the $\mathrm{ANU}^{1} 2.3 \mathrm{~m}$ diameter telescope based at the Siding Spring Observatory. The DBS is a general purpose optical spectrograph, permanently mounted at the Nasmyth A focus. The visible waveband is split by a dichroic at around $600 \mathrm{~nm}$ and feeds two essentially similar spectrographs, with red and blue optimised detectors respectively. The full slit length is 6.7 arcmin. Observations in the redder part (530 to $1000 \mathrm{~nm}$ ) are presented, with a 2-pixel resolution of $2 \AA$ or $7.5 \AA$.

\section{Previously known RCB stars}

38 spectroscopically confirmed RCB stars are known in our galaxy: $31 \mathrm{RCBs}^{2}$ were listed by Clayton (1996) and 7 others were recently discovered (Clayton et al. 2002; Hesselbach et al. 2003; Zaniewski et al. 2005). Fourteen of them lie in or near the area monitored by EROS-2.

Of the fourteen, eight are outside the EROS-2 fields: the newly active warm RCB V 2552 Ophiuchi (Hesselbach et al. 2003), V517 Oph, GU Sgr, V348 Sgr, WX Cra and 3 RCBs recently discovered by Zaniewski et al. (2005) in the MACHO database (MACHO-308.38099.66, MACHO-301.45783.9 that lies between 2 CCDs in the spiral arm field gs202, and MACHO401.48170.2237 between Bulge fields cg00841 and cg0051m).

The 6 others, which lie in the EROS-2 fields, are individually discussed here. Three of them (V739 Sgr, V3795 Sgr and VZ Sgr) were already listed as RCB stars by Zaniewski et al. (2005), but with wrong coordinates and thus also wrong MACHO identifiers. The correct values are listed in Table 1. We also indicate in this Table that three of those six RCB stars (V739 Sgr, VZ Sgr and MACHO-135.27132.51) showed large variations during the EROS-2 observation, but were not catalogued because they were either saturated or too faint on our reference images. For these stars, we then re-processed all our available EROS-2 data with our PSF photometry pipeline and

\footnotetext{
1 Australian National University.

2 We do not include V1773 Oph, GM Ser and V1405 Cyg as RCB stars as they have never been spectroscopically confirmed or shown photometric variations larger than 2.5 mag, see Kilkenny (1997) and Milone (1990).
}

new reference images to reconstruct their light curve. These reprocessed light curves are presented in Fig. 8.

- V739 Sgr: This star was reported as a possible RCB star by Lloyd Evans et al. (1991) due to its carbon spectrum and observed long-term photometric variability of large amplitude ( $\sim 4.5 \mathrm{mag}$ ). It has also been listed in the cool carbon star catalogue by Stephenson (1989). A smooth symmetric variation of 2.5 mag that lasts 120 days is observed in its MACHO light curve.

V739 Sgr was saturated on the EROS-2 reference images (subfield cg6245k) and has thus not been catalogued. Its reprocessed light curve shows 2 drops of $\sim 8$ mag (Fig. 8). These declines are characteristic of an RCB light curve.

- V3795 Sgr: This RCB star remained saturated in all EROS-2 epochs (subfield cg62731). Its AAVSO light curve show 2 declines, one before the EROS-2 observations (HJD $\simeq-1500$ ) and one after $(\mathrm{HJD} \simeq 2700)$, both larger than 3 mag.

The recovery phase of the first decline is also visible in the MACHO light curve, the second in the ASAS $-3^{3} V$ band light curve. We note that V3795 Sgr was saturated most of the time in the MACHO light curve, except for the portion below maximum light.

- VZ Sgr: The neighbourhood of this RCB star is very crowded. Its MACHO light curve shows a decline of 5 mag in about 20 days around JD-2 449 180. The MACHO curve consists of only 11 measurements, VZ Sgr being located in a low-sampling MACHO field.

VZ Sgr is also located in a low-sampling EROS-2 field $(\operatorname{cg} 0290 \mathrm{n})$ and was saturated on our reference images. Its reprocessed light curve shows variations of $\sim 6$ mag (Fig. 8). The MACHO and EROS variations can be better understood by comparison to the well sampled AAVSO light curve showing multiple declines since $\sim 2446000$.

- V1783 Sgr: This star was reported as a possible RCB star by Lloyd Evans et al. (1991) due to its H-poor carbon spectrum. It remained saturated in all EROS-2 epochs (located on subfield cg0842m). A slow decline $\left(\mathrm{d} V / \mathrm{d} t \sim 0.009 \mathrm{mag} \mathrm{day}^{-1}\right)$ occurred only a few days after the end of the EROS-2 observations (see its ASAS and AAVSO light curve).

- MACHO-135.27132.51: This RCB star, located in a lowsampling EROS-2 field (cg0353k), was too faint on our reference images and was thus also not catalogued. However, its re-processed light curve shows multiple variations of $\sim 8 \mathrm{mag}$ and one fast decline (Fig. 8).

- MACHO-118.18666.100: This star was properly catalogued and monitored in the EROS-2 database (named EROS2cg6075k26836). Its light curve is presented in Fig. 8. No drop in luminosity is observed, only some periodic oscillation features. Its classification as an RCB star being

\footnotetext{
3 ASAS: All Sky Automated Survey (Pojmanski 1997), URL: http://www . astrouw. edu.pl/ gp/asas/asas.html
} 


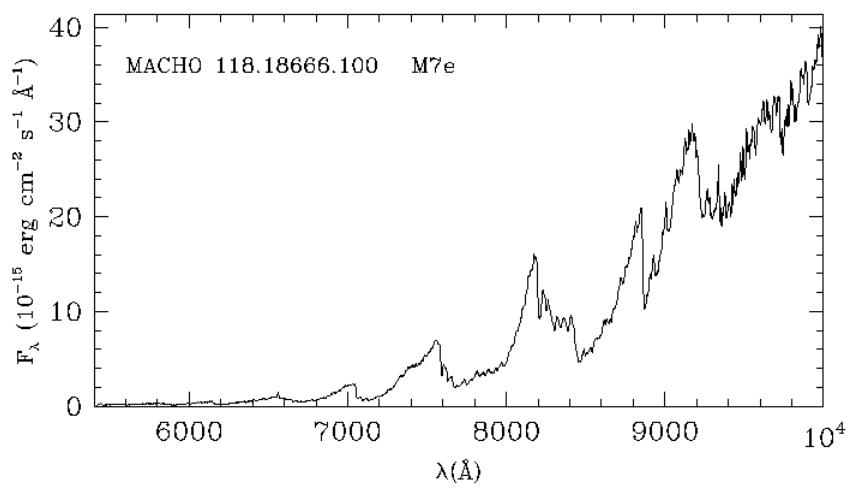

Fig. 1. Spectrum of MACHO-118.18666.100.

debatable, we took a spectrum during the night JD-2 454348. The spectrum, presented in Fig. 1, is typical of an M giant star, type M7e, with TiO features observable. Our spectrum is so different to the one presented by Zaniewski et al. (2005, Fig. 3) that, obviously, two different stars were observed. We are confident with our observation. We do not then support the classification of MACHO-118.18666.100 as an RCB star. Furthermore, we suggest that the $\sim 1.2 \mathrm{mag}$ variation in $\sim 150$ days observed in the MACHO light curve (Zaniewski et al. 2005, Fig. 1e) and the smaller one observed in the EROS2 light curve (see Fig. 8, JD 2451 900) are probably due to a sequence-D variation in a long secondary period (see Wood et al. 1999). A primary pulsation period, characteristic of such stars, is also seen.

In summary, six stars previously classified as RCBs are located in the EROS-2 fields; their status is summarized in Table 1. One of these stars is too faint and four others very bright and saturated in our reference images. Therefore, only one of the six was catalogued and monitored in the EROS-2 database, but its spectrum shows that it is not an RCB star, but an M giant. Overall, we failed to detect any of these known RCB stars. We also note that of those five, only three (MACHO-135.27132.51, VZ Sgr and V739 Sgr) showed detectable luminosity variations. Our light curve analysis would have detected them, had they been catalogued.

In view of this result, the completeness of our search is discussed in detail in Sect. 4.2.

\section{Mining the EROS-2 database}

The initial object catalogue used in the present analysis is an extended version of the cleaned EROS-2 source star catalogue produced by Hamadache et al. (2006) and Rahal (2003) for the microlensing analysis. We decided to enlarge our cleaning selection criteria for two reasons. First, over the period covered by the reference images, the RCB stars might be in a high luminosity phase, causing saturation in one band, or in a low luminosity phase, making the star undetectable in one band. Second, the low latitude fields are highly absorbed, making some RCBs undetectable in the blue band. Because of this, we decided to consider as valid all objects detected in at least one of the two filters. This strategy allows us to find RCBs that may have been catalogued in only one filter. Unfortunately, most of the new objects are due to light curves affected by diverse optical or electronic artefacts.

About $\sim 82$ and $\sim 25$ million different objects are in the extended catalogues of the Galactic Centre and spiral arm fields.
They all passed our simple artefact cleaning procedure that requires a minimum average flux of $300 \mathrm{ADU}\left(R_{\mathrm{E}} \sim 20.7\right.$ and $B_{\mathrm{E}} \sim 21.4$ ) and a minimum of 30 measurements per light curve.

Two different strategies, described in the following sections, have been used to find RCB stars in the two catalogues. For both strategies, we search for the outstanding characteristic of RCBs: a rapid drop in luminosity, by $\sim 2-8 \mathrm{mag}$. The first one is based on a series of selection cuts applied to each light curve, both filters being considered separately. The second is a visual inspection of the light curves of all EROS-2 objects catalogued either as a carbon star in the $\mathrm{CGCS}^{4}$ catalogue (Alksnis et al. 2001) and the Bulge carbon stars found by Azzopardi et al. (1991) or with a high infrared excess in the 2MASS database (Skrutskie et al. 2006). The second strategy was designed to verify the results of the first.

\subsection{Light curve analysis}

RCB optical light curves exhibit fast luminosity declines, at irregular intervals, followed by slow recoveries. Multiple consecutive fadings can be observed before the original brightness is recovered. Superimposed on these variations are intrinsic pulsations which produce low-amplitude periodic variations (for a good example, see the light curve of the R Coronae Borealis star prototype, R CrB, monitored since 1843 by the AAVSO ${ }^{5}$ ).

Guided by these properties, RCB candidates were selected by requiring that their light curves satisfy the 4 quantitative criteria given below. The light curves satisfying these criteria were then visually scanned to establish a list of spectroscopic targets.

The selection criteria were:

1. The difference between maximum and minimum flux, $F_{\max }$ and $F_{\min }$, must exceed $2.5 \mathrm{mag} . F_{\max }$ and $F_{\min }$ are determined as the averages of the 10 extreme values after eliminating the five highest and lowest flux points.

2. The maximum flux must be higher than 5000 ADU, equivalent to a magnitude at maximum of about 18 (here, the maximum flux is averaged over 10 consecutive points).

3. The third selection criterion was designed to reject most short-period variable stars. We retain objects whose light curve crosses the median flux $F_{\text {med }}=1 / 2 \times\left(F_{\max }+F_{\min }\right)$ by at least 3 consecutive points, less than 12 times.

4. After application of the first three selection criteria, 28391 objects remained in the Bulge fields and 15731 in the spiral arm fields. The light curves of these objects are mainly due to artefacts and show irregular/erratic variations. A fourth criterion was therefore designed to eliminate light curves dominated by artefacts, based on the ratio $R$ between the point-to-point dispersion of the light curve, $\sigma_{\text {ptp }}$, and the median flux, $F_{\text {med }}$. Here, $\sigma_{\text {ptp }}$ is defined as the dispersion of the flux of individual points from the flux obtained by linear interpolation between the two neighbouring points. Figure 2 illustrates the cuts applied in the plane of $\sigma_{\mathrm{ptp}} / F_{\text {med }}$ vs. $\log F_{\text {med }}$ to remove artefact-dominated light curves, based on the locus of a random sample of "normal" stars.

The remaining 8524 stars (7181 in the cg field and 1343 in gs, gn, tm and bs, see Table 2) are mainly Mira and other LongPeriod Variables. 23 of these stars were selected as RCB candidates by visual inspection, based on the main characteristic

\footnotetext{
${ }^{4}$ CGCS: the Catalogue of Galactic Cool Carbon Stars, including 6891 entries collected from the literature.

5 American Association of Variable Star Observers, URL: http:// wWw . aavso.org/vstar/vsots/0100.shtml
} 


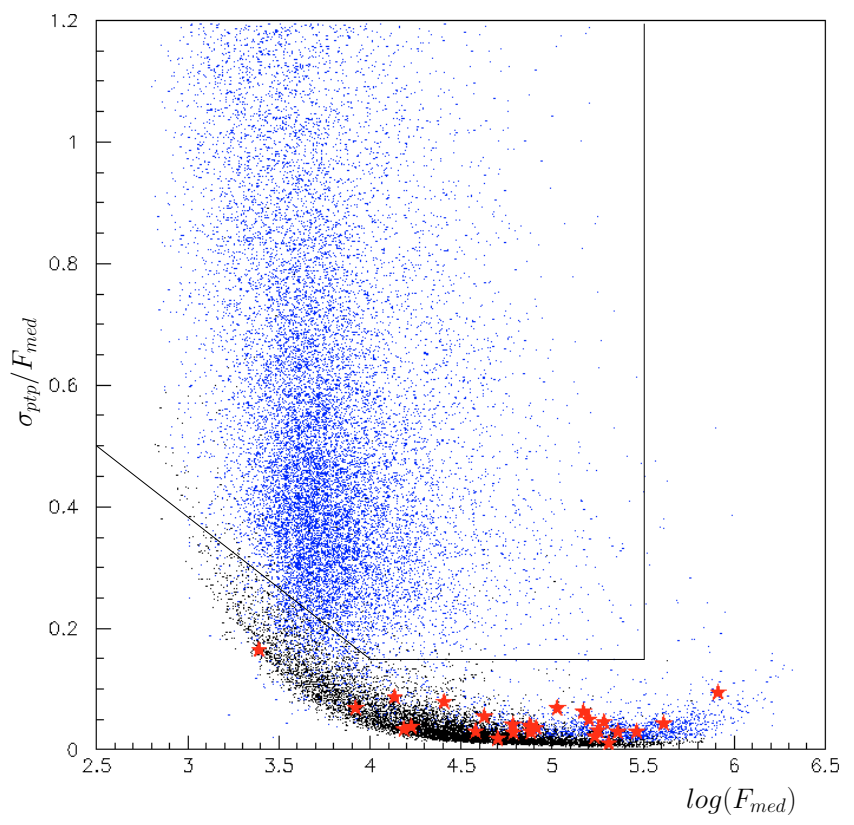

Fig. 2. Ratio $R\left(=\sigma_{\text {ptp }} / F_{\text {med }}\right)$ vs. median magnitude $F_{\text {med }}$. The blue dots show the 28391 Bulge and 15731 spiral arm objects that pass cuts 1 , 2 and 3. The black dots are random stars from one of our sky areas; they show significantly lower point-to-point dispersion at given $F_{\text {med }}$. We retain only objects below or to the right of the straight lines. The 28 red stars show the position of the 14 new RCBs in both filters.

of RCB stars, a rapid and non-periodic drop in luminosity. But because our irregular sampling may mislead our interpretation, we decided to use loose visual selection criteria. Thus, not all 23 RCB candidates have light curves with a large, rapid drop in luminosity. Half of them have only an infra-red excess $(J-K>1)$ and large variations with no clear indication of periodicity, so their classification as RCB stars was considered uncertain. Hence, spectroscopy was obtained for these 23 stars, with results as described in Sect. 4.4.

\subsection{Detection efficiency}

The unpredictable nature of RCB light curves, our survey characteristics, and the interstellar extinction influence the results of our search. We discuss here how it affects our detection efficiency analysis.

\subsubsection{Influence of the interstellar extinction}

Without dust extinction along our lines of sight, RCB stars closer than $\sim 16, \sim 12, \sim 8.8$ and $\sim 5.8 \mathrm{kpc}$ for RCB absolute magnitudes $M_{V}$ of $-5,-4,-3$ and -2 , respectively, would not be detected by EROS-2, due to saturation of the detectors, assuming a distance modulus of 14.4 for the Galactic centre, corresponding to $R_{0}=7.6 \pm 0.3 \mathrm{kpc}$ (Eisenhauer et al. 2005). In our fields at low Galactic latitude $\left(|b|<8^{\circ}\right.$, see Fig. 3), the concentration of dust is high, and the photometry of the new RCB stars is most likely affected by extinction. As extinction increases with decreasing Galactic latitude, their detection depends on the field monitored.

As most known RCB stars have $-5<M_{V}<-3$, most RCB candidates in the Bulge would thus be saturated (and therefore undetected) in EROS-2 images unless extinction is significant. However, our fields with $|b|<5^{\circ}$ have sufficient absorption to ensure that Bulge RCB with $M_{V}>-5$ are not saturated (see Sect. 5.2). Our detection efficiency is therefore independent of the absolute magnitude of Bulge RCBs in these fields, which constitute about $75 \%\left(\sim 58 \mathrm{deg}^{2}, 61\right.$ fields $)$ of the total Bulge area monitored.

For $|b|>5^{\circ}$, bright RCBs would be saturated, but only $25 \%$ of the EROS-2 fields are in this zone. We note the possibility that these bright RCBs could not be saturated on our reference images if they are in a declining phase, but it is less likely as the reference images are built with 15 images, distributed over time. These $25 \%$ fields will not be used to measure the RCB scale height in Sect. 5.4. As the minimum extinction observed in those fields is $A_{B_{\mathrm{E}}} \sim 0.7 \mathrm{mag}$, the detection loss due to saturation will not affect Bulge RCB stars fainter than $M_{V} \lesssim-3.7$.

\subsubsection{Influence of the survey characteristics}

Two main factors have to be considered in estimating the proportion of RCB stars that are detectable in our survey. First, since the EROS-2 monitoring period of 6.7 years is finite, RCB stars can remain bright or faint throughout the entire observing period and thus be missed by our detection algorithm, which targets only large luminosity drops ( $>2.5 \mathrm{mag}$ ). The evaluation of this effect is complicated by the fact that the frequency of fadings varies from one RCB to another by a factor $\sim 100$ (see Jurcsik 1996), this frequency being strongly correlated to the $[\mathrm{C} / \mathrm{H}]$ abundance ratio. Second, potential luminosity drops can also remain undetected because our light curve analysis is affected by the observational sampling. We tried to evaluate the effect of these factors by studying the behaviour of known RCB stars and by simulating synthetic RCB light curves. The details of the studies are as follows.

- We estimate the average probability that an RCB star would not have a luminosity drop larger than $2.5 \mathrm{mag}$ during the 6.7-year observing period. We know that 3 of the 5 known Galactic RCB stars located in our fields $(60 \%)$ remained bright during that period (see Sect. 3). However, we found a larger value of $\sim 94 \%$ by studying the AAVSO light curves of two well known RCB stars, R CrB and RY Sgr, which have been monitored for more than a century with a median sampling of $\sim 0.3$ day. This last value corresponds to the probability that a survey with a time window identical to that of EROS-2 will not encounter a drop in brightness of more than $2.5 \mathrm{mag}$. But as those two RCB stars might not be representative, but have different dust production rates and thus drop in luminosity with different frequencies, we used the value estimated by Alcock et al. (2001) based on a larger sample. They found than only 23 of 31 known Galactic RCB stars monitored by AAVSO had experienced at least one decline deeper than 3 mag during the 7 years of their observation. It corresponds to a probability of $\sim 75 \%$, which is also an intermediate value between the two previous ones.

- RCB stars could also stay non-detectable in a faint phase during all the EROS-2 observation, but it is difficult to estimate the probability of this occurring. We know that $\mathrm{R} \mathrm{CrB}$ and RY Sgr have never shown a faint phase that lasted longer than 3.3 years. However, most of the known RCBs could be only the tip of the iceberg, as higher dust production could be a more general case. For example, the SMC star MSX-SMC014 was recently found to be an RCB candidate by Kraemer et al. (2005) from its infrared spectral characteristics, but we found no optical counterpart in the EROS-2 database and also no brightness variation during the 6.7 years observation (EROS-2 SMC magnitude limit: $B_{\mathrm{E}}>22.4$ and $R_{\mathrm{E}}>21.7$ ). Another good example is the RCB star V854 Cen. It is the 


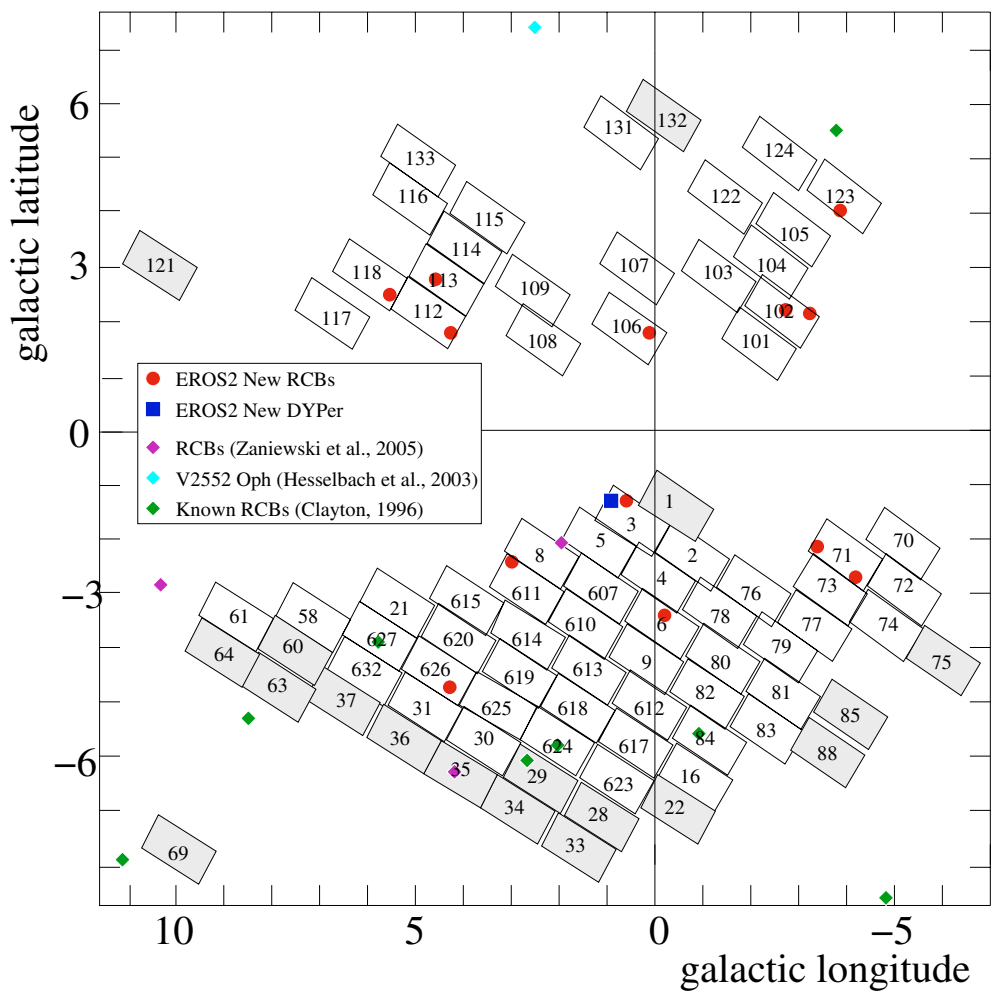

Fig. 3. Map of the 83 EROS-2 Bulge fields in Galactic coordinates. The 18 less frequently observed fields are coloured grey. The location of the newly confirmed Galactic RCBs stars are indicated along with the four discovered by Zaniewski et al. (2005), the newly active warm RCB V 2552 Ophiuchi (Hesselbach et al. 2003) and the other previously known RCBs in this region (V1783 Sgr, V739 Sgr, V3795 Sgr, VZ Sgr, V517 Oph, Gu Sgr, V348 Sgr and WX Cra). 3rd brightest RCB star in the sky at present but it was generally fainter than 13th magnitude and no brighter than 10th from 1913 to 1952 (Clayton 1996).

- If an RCB star is too faint on our reference images, it will not be catalogued and thus detected. As 15 images, distributed in time, were used to build the reference image, the chance of missing an RCB star at that step is low. Of the 17 LMC RCB stars listed by Alcock et al. (2001), 16 were located in our fields and all were catalogued. On the 6 known Galactic RCB stars, only one was too faint (see Sect. 3). Therefore, from those 22 known RCB stars we estimate our efficiency at the catalogue step to be $\sim 95 \%$.

- The number of measurements in the light curve of a star varies depending on its position on the CCD. Stars located on the edge have fewer measurements due to the pointing accuracy.

To evaluate the influence of the real observational sampling on our detection efficiency, we used a Monte Carlo simulation of light curves. We added 1 to 3 brightness drops to simulated light curves of bright stars $\left(B_{\mathrm{E}}=13\right)$ with epochs identical to the fields and distributed in each of the monitored sky areas. Each luminosity drop imitates those observed in known RCB stars and has three consecutive phases: a steep decline at a constant rate of +0.045 mag day $^{-1}$, corresponding to the average for all the new Galactic RCBs, see Table 7); then a plateau and finally a recovery to the original magnitude at a rate of -0.02 mag day $^{-1}$, slower than the rate of the decline. The simulation has three randomly generated parameters: the date at the start of the decline (chosen to be during the EROS-2 observations); the duration of the plateau (from 10 to 300 days); and its depth (from 2.5 to $8 \mathrm{mag}$ ). The light curves created were then subjected to the same search pipeline as the real ones. The effect of the last selection criteria, the visual examination, was estimated by looking at one hundred simulated light curves, randomly selected. We estimate that the visual selection could not affect the final detection efficiency by more than $5 \%$. Overall, this simple simulation showed that our detection efficiency for obscured RCB stars is excellent. On average, it exceeds 94\% for the 112 main sky areas monitored (Galactic centre and spiral arms) and drops to $\sim 45 \%$ for the 18 less frequently observed fields (they are shown in Fig. 3).

In summary, the detection of RCB stars should be of the order of $\sim 60 \%(0.75 \times 0.95 \times 0.94)$ complete for the majority of our survey $(\sim 80 \%)$. Some infrequently observed fields may be less complete in RCB star detection.

\subsection{Visual inspection of pre-selected catalogued stars}

As a check of the light curve analysis, we inspected the light curves of all stars in our EROS-2 fields that match two characteristics of RCB stars: a carbon star spectrum and an infrared excess.

Therefore, we inspected the light curves of all stars listed in the carbon star catalogue (Alksnis et al. 2001) and showing an infrared excess $(J-K>1)$, the Bulge carbon stars found by Azzopardi et al. (1991) and objects in the 2MASS catalogue with a high infrared excess (see the selection area delimited in Fig. 5). 35 carbon stars and 2082 2MASS objects (with an EROS-2 matched object within 2 arcsec) were inspected. The visual inspection resulted in the rediscovery of 10 RCBs already detected by the light curve analysis, but no new RCB stars appeared. This supports the efficiency of our first analysis. The rediscovered RCBs show a high infrared excess in Fig. 5 since the 2MASS epochs for nine of them happened to be, by chance, either in a faint or declining/recovering phase.

\subsection{Spectroscopic selection}

If a well-sampled light curve is available, identification with the RCB class can be made with fairly high confidence because of 

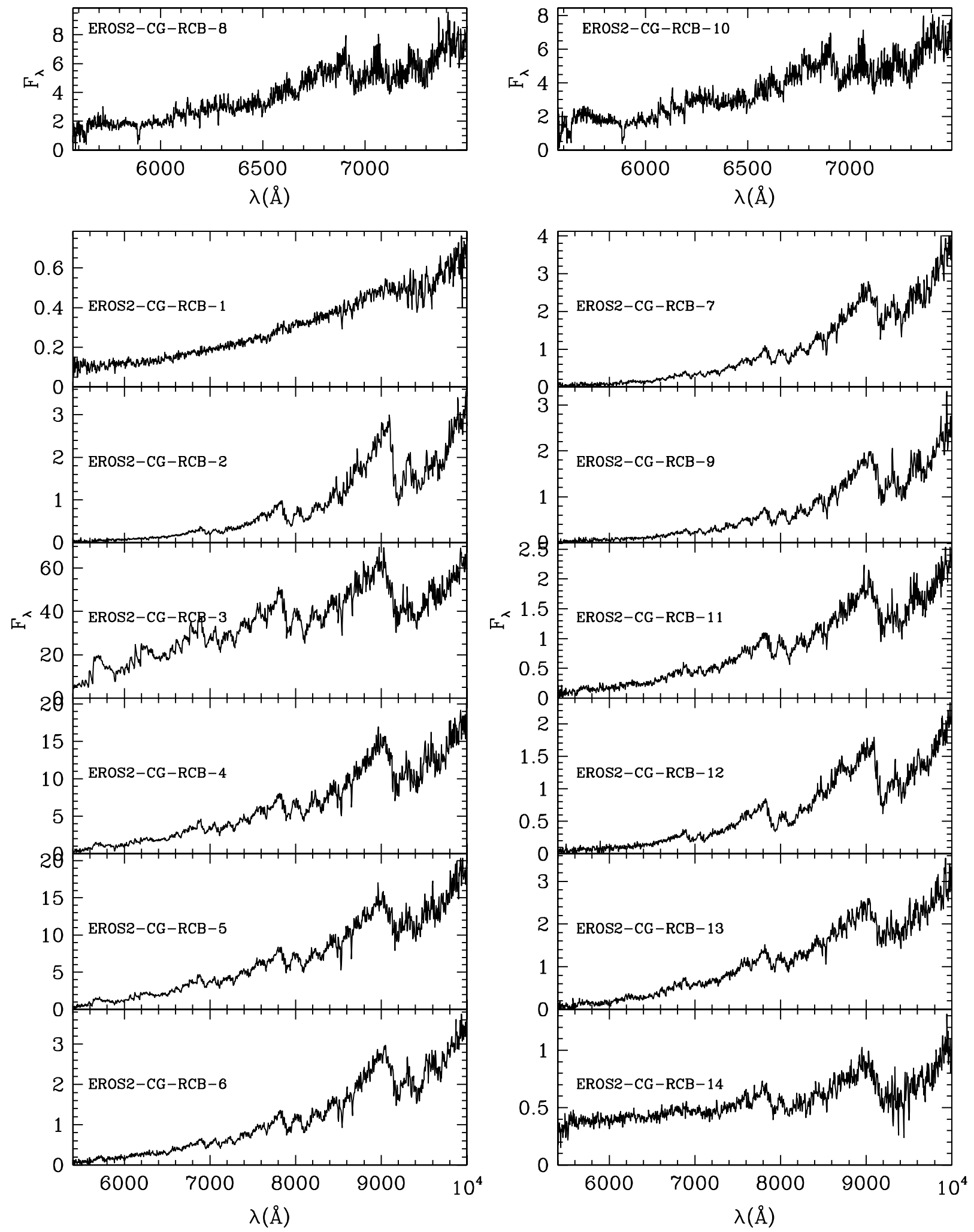

Fig. 4. Spectra of the RCB star candidates. $F_{\lambda}$ is in units of $10^{-15} \mathrm{erg} \mathrm{cm}^{-2} \mathrm{~s}^{-1} \AA^{-1}$. The top two spectra have a resolution of $2 \AA$ while the bottom 12 spectra have a resolution of $7.5 \AA$. No correction for interstellar reddening has been applied.

the distinct nature of the RCB brightness drops. Of the 23 candidates, half showed a drop of less than 4 mag and were thus not strong RCB candidates. Spectroscopic information was necessary to reveal their real nature.

Twenty one candidates were observed during the nights JD-2 454215 and JD-2 454348; the last two were too faint (cg0334k19434, cg6271119693). Of the 21, 13 have spectra with carbon features due to $\mathrm{C}_{2}$ and/or CN molecules (see Fig. 4) and are therefore considered as confirmed new R Coronae Borealis stars. In addition to these 13 stars, we retain one other RCB candidate (cg003013740), because its light curve has multiple declines of more than 8 mag. The spectrum of this star (Fig. 4) is 
Table 3. General information on the new Galactic RCB stars.

\begin{tabular}{|c|c|c|c|c|}
\hline $\begin{array}{l}\text { EROS2 Galactic } \\
\text { RCB name }\end{array}$ & $\begin{array}{l}\text { EROS2 star } \\
\text { Identifier }\end{array}$ & "Coordinates $\left(\mathrm{J}_{2000}\right)$ & Other Id & General information \\
\hline EROS2-CG-RCB-1* & $\operatorname{cg} 003013740$ & $17: 52: 19.96-29: 03: 30.8$ & & \\
\hline EROS2-CG-RCB-2^ ${ }^{\star}$ & $\operatorname{cg} 0030 \mathrm{~m} 154$ & $17: 52: 48.70-28: 45: 18.9$ & & DY Per candidate \\
\hline EROS2-CG-RCB-3* & $\operatorname{cg} 0062118324$ & $17: 58: 28.27-30: 51: 16.4$ & & just outside the OGLE2 field SC23 \\
\hline EROS2-CG-RCB-4* & $\operatorname{cg} 0711 \mathrm{~m} 12518$ & $17: 46: 16.20-32: 57: 40.9$ & Terz V $2046^{a}$ & \\
\hline EROS2-CG-RCB-5 ${ }^{\star}$ & $\operatorname{cg} 0715 n 14430$ & $17: 46: 00.32-33: 47: 56.6$ & & \\
\hline EROS2-CG-RCB-6 ${ }^{\star}$ & $\operatorname{cg} 1024 \mathrm{~m} 18795$ & $17: 30: 23.83-30: 08: 28.3$ & V1135 Sco ${ }^{b}$ & \\
\hline EROS2-CG-RCB-7^ & $\operatorname{cg} 1026113692$ & $17: 29: 37.09-30: 39: 36.7$ & Terz V $1680^{a}$ & \\
\hline EROS2-CG-RCB-8*夫 & $\operatorname{cg} 1066 \mathrm{~m} 15039$ & $17: 39: 20.72-27: 57: 22.4$ & Terz V $2960^{c}$ & \\
\hline EROS2-CG-RCB-9 & $\operatorname{cg} 1127113076$ & $17: 48: 30.87-24: 22: 56.5$ & & \\
\hline EROS2-CG-RCB-10^^ & cg1133k13881 & $17: 45: 31.41-23: 32: 24.4$ & & \\
\hline EROS2-CG-RCB-11* & $\operatorname{cg} 1187116577$ & $17: 48: 41.53-23: 00: 26.5$ & & just outside the OGLE2 field SC15 \\
\hline EROS2-CG-RCB-12 ${ }^{\star}$ & $\operatorname{cg} 1235 \mathrm{~m} 14181$ & $17: 19: 58.50-30: 04: 21.3$ & & \\
\hline EROS2-CG-RCB-13* & cg6110k17996 & $18: 01: 58.22-27: 36: 48.3$ & МACHO-176.19607.1138 & \\
\hline EROS2-CG-RCB-14* & $\operatorname{cg} 6267 k 28863$ & $18: 13: 14.86-27: 49: 40.9$ & & \\
\hline
\end{tabular}

${ }^{a}$ Terzan \& Ounnas (1988), ${ }^{b}$ Kukarkin et al. (1971), ${ }^{c}$ Terzan \& Gosset (1991).

` Spectral resolution $7.5 \AA$ A per pixel. ${ }^{\star \star}$ Resolution 2.0 Å per pixel.

Table 4. General information on the rejected candidates.

\begin{tabular}{|c|c|c|c|c|}
\hline $\begin{array}{l}\text { EROS2 star } \\
\text { Identifier }\end{array}$ & $\overline{\text { Coordinates }\left(\mathrm{J}_{2000}\right)}$ & Classification & Other Id & General information \\
\hline cg0334k19434 & $18: 18: 15.10-31: 51: 54.6$ & AM Her & MACHO-155.26433.492 & \\
\hline $\operatorname{cg} 0606 \mathrm{k} 27985^{\star \star}$ & $18: 16: 54.22-25: 02: 54.7$ & M5 & МАCHO-177.26016.56 & \\
\hline $\operatorname{cg} 1047 \mathrm{k} 14026^{\star}$ & $17: 28: 07.82-29: 48: 14.0$ & M5 & & \\
\hline $\operatorname{cg} 1076 \mathrm{k} 13424^{\star}$ & $17: 33: 26.21-27: 32: 02.7$ & M2 & & suddenly appears 3 mag brighter \\
\hline $\operatorname{cg} 1077 \mathrm{~m} 26188^{\star}$ & $17: 36: 15.13-27: 37: 11.4$ & M6 & & periodic? \\
\hline $\operatorname{cg} 1131 \mathrm{n} 13463^{\star \star}$ & $17: 46: 00.16-23: 21: 16.1$ & M0 & ASAS174600-2321.3 ${ }^{a}$ & drop of 2.5 mag in ASAS light curve \\
\hline $\operatorname{cg} 1135 \mathrm{~m} 3139^{\star}$ & $17: 46: 12.87-23: 49: 44.2$ & M3 & & \\
\hline $\operatorname{cg} 6126 \mathrm{~m} 5824^{\star}$ & $18: 05: 30.42-31: 53: 24.2$ & M2 & & \\
\hline $\operatorname{cg} 6271119693$ & $18: 13: 16.97-25: 31: 34.8$ & M? & & \\
\hline
\end{tabular}

${ }^{a}$ ASAS: All Sky Automated Survey (Pojmanski 1997).

^ Spectral resolution 7.5 A per pixel. ${ }^{\star \star}$ Resolution 2.0 Å per pixel.

almost totally featureless, although some very weak $\mathrm{CN}$ bands are present $(\lambda \sim 7876 \AA$ and $9100 \AA)$. The star was very faint during the observation. The identification numbers, positions, and alternative names for our final sample of 14 new RCB star are given in Table 3 .

One of these 14 new RCB stars, EROS2-RCB-CG-2, has a light curve that resembles that of the unusual Galactic RCB star DY Per (Alksnis 1994). Its light curve shows declines of $>4$ mag. that are slower than those of most RCB stars. The declines are also associated with more symmetric recoveries.

The remaining 7 stars show M-type spectra, corresponding to oxygen-rich red giants. They were therefore rejected as RCB stars, even though their light curves show sudden drops in luminosity by up to $3.5 \mathrm{mag}$. In addition, we did not keep the 2 objects that were too faint for spectroscopy: the star cg6271119693 has a light curve that resembles those of the M-type red giants, and cg0334k19434 has a light curve which is more like that of an AM Her star. The identifications and positions of these 9 stars are listed in Table 4.

\subsubsection{Radial velocities of confirmed RCBs}

Radial velocities were obtained by cross-correlating spectra obtained at resolutions of $17,45,72$ or $151 \mathrm{~km} \mathrm{~s}^{-1} /$ pixel during the night JD-2 454348, using the spectrum of the carbon star $\mathrm{X}$ Vel as a template. The radial velocity of $\mathrm{X}$ Vel was taken to be $-5.4 \mathrm{~km} \mathrm{~s}^{-1}$ (determined from high resolution echelle spectra which were cross-correlated against the radial velocity standard $\alpha$ Cet with a velocity of $-25.8 \mathrm{~km} \mathrm{~s}^{-1}$ ). The heliocentric radial velocities and their errors are listed in Table 5. We did not measure the radial velocity of RCB-1 as its spectrum shows no carbon features.

The measured radial velocities cover a large range, from -325 to $115 \mathrm{~km} \mathrm{~s}^{-1}$, with no convincing trend with Galactic latitude. This large range of values is consistent with the radial velocities found for OH/IR stars, oxygen-rich evolved stars, located in the Galactic Bulge at that particular Galactic longitude (see Sevenster 1999, Fig. 12).

\subsection{2. ${ }^{13} \mathrm{C}$ test and $\mathrm{Ca}$ II triplet}

The absence of enhanced ${ }^{13} \mathrm{C}$ is a characteristic of RCB stars (Pollard et al. 1994). We tried to estimate the abundance of ${ }^{13} \mathrm{C}$ using ${ }^{13} \mathrm{CN}$ band at $6260 \AA$ (see Lloyd Evans 1991). It was possible to determine the absence of a significative presence of ${ }^{13} \mathrm{C}$ for seven RCBs of the 14 new RCBs. The spectra of these 7 RCBs are higher resolution $(2 \AA)$. For the remaining $\mathrm{RCBs}$, the low resolution spectra and the small signal due to extinction prevent us from determining the abundance of ${ }^{13} \mathrm{C}$. The results are summarized in Table 5.

An interesting feature in the spectrum of warm carbon stars is the infrared triplet due to ionised calcium $(\lambda \sim 8498,8543$ and $8662 \AA$ ). Richer (1971) shows that the intensity of those lines is a good indicator of the carbon star temperature; the cooler the 
Table 5. Heliocentric radial velocities and ${ }^{13} \mathrm{C}$ presence.

\begin{tabular}{lccccc}
\hline \hline RCB & $\begin{array}{c}l \\
(\mathrm{deg})\end{array}$ & $\begin{array}{c}b \\
(\mathrm{deg})\end{array}$ & $\begin{array}{c}V_{\text {hel }} \\
\left(\mathrm{km} \mathrm{s}^{-1}\right)\end{array}$ & $\begin{array}{c}\sigma_{V} \\
\left(\mathrm{~km} \mathrm{~s}^{-1}\right)\end{array}$ & $\begin{array}{c}{ }^{13} \mathrm{C} \\
\text { presence? }\end{array}$ \\
\hline 1 & 0.65107 & -1.32119 & - & - & - \\
2 & 0.96560 & -1.25721 & 68.4 & 13.7 & - \\
3 & -0.23313 & -3.37369 & 46.9 & 2.6 & no \\
4 & -3.3663 & -2.21249 & -246.4 & 3.1 & no \\
5 & -4.11175 & -2.59990 & -323.1 & 0.7 & no \\
6 & -2.79018 & 2.15220 & 114.2 & 3.5 & no \\
7 & -3.31629 & 2.00684 & -111.4 & 6.8 & - \\
8 & 0.10674 & 1.68854 & 111.9 & 3.7 & no \\
9 & 4.23037 & 1.80687 & 112.5 & 1.0 & - \\
10 & 4.59793 & 2.82737 & 76.4 & 3.8 & no \\
11 & 5.43103 & 2.47977 & 73.0 & 4.4 & no \\
12 & -3.99599 & 4.05891 & -43.7 & 5.4 & - \\
13 & 2.96514 & -2.43305 & -292.1 & 0.9 & - \\
14 & 3.98548 & -4.72163 & -11.4 & 0.8 & - \\
\hline
\end{tabular}

temperature, the weaker the lines. In the spectra presented in Fig. 4, the Ca II feature was observable only in the case of low resolution spectra. On these 12 spectra, 10 present either strong or moderate $\mathrm{Ca}$ II lines and two have no or very weak $\mathrm{Ca}$ II lines (EROS2-CG-RCB-2 and -12). These 2 RCBs are therefore intrinsically cooler than the others. We note that EROS2-CGRCB-2 is a DY Per RCB, which are known to be cool RCBs. This is important as all spectra are strongly reddened, the stars being close to the Galactic plane. This temperature indication will be use in Sect. 5.2 as a check of our extinction correction.

\section{The new R Coronae Borealis stars}

None of the newly discovered RCB stars in Table 3 is located in the spiral arm fields; they are all found in the direction of the Galactic Bulge, distributed equally between the north and south sides of the Galactic plane. Their positions are shown on the Bulge field map in Fig. 3, finding charts in Fig. 10, and the light curves in Figs. 9-9. The actual measurements are available at http://eros.in2p3.fr/Variables/ RCB/RCB-CGBS . html

Only one of the 14 new RCB stars, EROS2-CG-RCB13 , is located in the MACHO fields (MACHO identifier 176.19607.1138). None is located in the OGLE-II fields.

\subsection{Infrared properties}

Most RCB stars have a near-IR excess even if they have not shown a decline for several years. Dust can still form continuously near the star, without completely obscuring its photosphere (Feast 1997). JHK measurements have been made by the 2MASS project for all the new Galactic RCBs (see Table 6), and all have $J-K>1.9$, indicative of an infrared excess (see Fig. 5). Part of this excess is due to the high interstellar extinction, but we note also that the 2MASS epochs for 10 of the $14 \mathrm{RCBs}$ coincide with a faint or dimming/recovering phase.

EROS2-CG-RCB-2 is in the region of Fig. 5 occupied by common carbon stars: this area also corresponds to the typical position for DY Per-type RCB stars. This result, along with the DY Per-like light curve, confirms the membership of this star in the DY Per class of RCB stars.

We note that all new RCB stars have entries in the MSX6C Infrared Point Source Catalog (Egan et al. 2003), except the DY Per-like RCB EROS2-CG-RCB-2 and EROS2-CG-RCB-12.

\subsection{Extinction correction and distances}

If all new RCBs are in the Bulge, then their apparent luminosity is strongly affected by reddening. The extinction towards small areas of the Bulge can be determined from the position of the red-giant clump in the colour-magnitude diagram. The selection of clump-giant samples was performed for each quarterCCD subfield (about $10.3^{\prime} \times 10.3^{\prime}$ ) as described in Hamadache et al. (2006). The result is presented in the top panel of Fig. 6, where the expected linear dependence between the fitted magnitudes $B_{\mathrm{E} \text {,clump }}$ and the colours $\left\langle B_{\mathrm{E}}-R_{\mathrm{E}}\right\rangle_{\text {clump }}$ of the clump centre for different samples is shown. The all-sky COBE/DIRBE extinction map by Schlegel et al. (1998) was not used to estimate reddening because it overestimates the amount of dust in highextinction areas (Stanek 1998; Arce \& Goodman 1999; Dutra et al. 2003). The lower panel of Fig. 6 confirms the non-linear dependence between the reddening $E(B-V)$ from Schlegel et al. (1998) and the reddening $\left\langle B_{\mathrm{E}}-R_{\mathrm{E}}\right\rangle_{\text {clump }}$ derived here. However, the lower panel of Fig. 6 can be used to define the EROS-2 zeroextinction colour of the Bulge red clump giant centre. We find $\left(B_{\mathrm{E}}-R_{\mathrm{E}}\right)_{0, \text { clump }} \approx 0.6$. Our intrinsic clump colour, when transformed by Eq. (1), is close to the expected colour $\langle V-I\rangle_{0, \text { clump }} \sim$ 1.0 for the giant clump in the Bulge (Sumi 2004).

From Fig. 6, we obtained the following visual extinction law:

$A_{B_{\mathrm{E}}}=-1.77+2.95\left\langle B_{\mathrm{E}}-R_{\mathrm{E}}\right\rangle_{\text {clump }}$.

From this relation, we estimated the extinction to each RCB star based on the colour of clump-giant stars located within 3 arcmin. The correction applied to each RCB star colour and magnitude at maximum is given on Table 7 .

The positions of the de-reddened RCB stars are shown in the $M_{V}$ vs. $V-I$ diagram, Fig. 7 , and the derived intrinsic values, $M_{V}$ and $(V-I)_{0}$, are given in Table 7 . The $V$ and $I$ magnitudes were obtained from $B_{\mathrm{E}}$ and $R_{\mathrm{E}}$ using Eq. (1). The positions of 10 (resp. 3) common $\mathrm{RCBs}^{6}$ and 4 (resp. 2) DY Per-like RCBs in the LMC (resp. SMC) (Alcock et al. 2001; Tisserand et al. 2004) are also shown in Fig. 7. We used the EROS-2 light curve to measure the maximum brightness of each RCB star. The distance modulus used was 14.4 mag for the Galactic Centre (the present best measure of the galactocentric distance is $R_{0}=7.6 \pm 0.3 \mathrm{kpc}$, see Eisenhauer et al. 2005), $18.5 \mathrm{mag}$ for the LMC and $18.9 \mathrm{mag}$ for the SMC. We also corrected the LMC and SMC magnitudes for the total reddening (Galactic foreground + intrinsic dust), corresponding to $E(B-V)_{\mathrm{LMC}} \sim 0.17$ and $A_{V, \mathrm{LMC}} \sim 0.5 \mathrm{mag}$ for the LMC (Alcock et al. 2001), $E(B-V)_{\mathrm{SMC}} \sim 0.06$ and $A_{V, \mathrm{SMC}} \sim 0.17 \mathrm{mag}$ for the SMC (Zaritsky et al. 2002).

The fact that the Bulge reddening correction results in an overlap in Fig. 7 between Galactic and Magellanic Cloud RCB stars suggests that most of the new RCB stars are indeed in the Galactic Bulge (the Bulge occupies a region between 6 and $10 \mathrm{kpc}$ ). Note that without the reddening correction, the new Galactic RCB stars would be the coolest RCBs known $\left(T_{\text {eff }}<3600 \mathrm{~K}\right.$, with a median at $\left.\sim 3000 \mathrm{~K}\right)$. With the reddening correction, the effective temperatures of most of the new RCB stars are as expected for RCB stars. Most currently known RCB stars are carbon-rich supergiants with an effective temperature between 5000 and $7000 \mathrm{~K}$ (Clayton 1996), which is true also for the RCB stars in the LMC.

Even with the Bulge extinction correction, four of the new Galactic RCBs (EROS2-CG-RCB-5, -9, -12 and -14) are cooler

\footnotetext{
6 Three LMC RCB stars were not used: MACHO-6.6575.13 which never reached its maximum brightness in the EROS-2 data, HV 12842 which is outside the EROS-2 fields, and the hot RCB star MACHO11.8632 .2507 .
} 
Table 6. Near-IR photometry.

\begin{tabular}{|c|c|c|c|c|c|c|c|c|}
\hline RCB name & JD Epoch 2MASS & $J_{2 \mathrm{MASS}}$ & $H_{2 \mathrm{MASS}}$ & $K_{2 \mathrm{MASS}}$ & JD Epoch Denis & $I_{\text {DENIS }}$ & $\overline{J_{\text {DENIS }}}$ & $K_{\text {DENIS }}$ \\
\hline EROS2-CG-RCB-1 & $2451010.6571^{\star}$ & 12.502 & 10.845 & 9.077 & $2451404.57740^{\star}$ & 14.582 & 13.635 & 9.615 \\
\hline EROS2-CG-RCB-2 & $2451010.6591^{\vee}$ & 11.389 & 10.061 & 9.290 & $2451410.59416^{\wedge}$ & 14.394 & 11.223 & 9.197 \\
\hline EROS2-CG-RCB-3 & $2451040.5447^{\star}$ & 12.702 & 10.348 & 8.538 & & & & \\
\hline EROS2-CG-RCB-4 & $2451039.6137^{\diamond}$ & 10.312 & 9.087 & 7.936 & & & & \\
\hline EROS2-CG-RCB-5 & $2451039.6095^{\star}$ & 15.524 & 12.715 & 9.977 & & & & \\
\hline EROS2-CG-RCB-6 & $2451035.6283^{\diamond}$ & 10.193 & 9.151 & 8.277 & $2450313.53086^{?}$ & & 13.882 & 9.487 \\
\hline EROS2-CG-RCB-7 & $2451035.6272^{\vee}$ & 11.806 & 10.310 & 9.061 & $2450216.79936^{?}$ & & 14.755 & 10.494 \\
\hline & & & & & $2450313.53012^{?}$ & & 14.264 & 9.856 \\
\hline EROS2-CG-RCB-8 & $2450996.7036^{\star}$ & 13.152 & 11.518 & 9.895 & & & & \\
\hline EROS2-CG-RCB-9 & $2451010.6160^{\vee}$ & 12.458 & 10.484 & 8.808 & $2451074.74752^{\vee}$ & 16.633 & 12.509 & 8.854 \\
\hline EROS2-CG-RCB-10 & $2450963.8322^{\wedge}$ & 11.117 & 9.534 & 7.975 & $2451103.66233^{\diamond}$ & & 14.878 & 9.202 \\
\hline EROS2-CG-RCB-11 & $2450950.8589^{\diamond}$ & 10.106 & 9.059 & 8.109 & $2451074.74555^{\diamond}$ & 12.535 & 10.345 & 8.183 \\
\hline EROS2-CG-RCB-12 & $2451035.5272^{\star}$ & 13.848 & 11.949 & 10.425 & $2451381.63688^{\star}$ & 16.687 & 12.951 & 9.876 \\
\hline EROS2-CG-RCB-13 & $2451364.6757^{\star}$ & 13.203 & 11.330 & 9.490 & $2450174.90133^{\star}$ & 15.374 & 13.393 & 9.699 \\
\hline EROS2-CG-RCB-14 & $2451749.4873^{\diamond}$ & 11.347 & 9.807 & 8.340 & & & & \\
\hline
\end{tabular}

$\star$ : During a faint phase, $\diamond$ : during a bright phase, $\vee$ and $\wedge$ : during a dimming or recovering phase, ?: phase unknown.

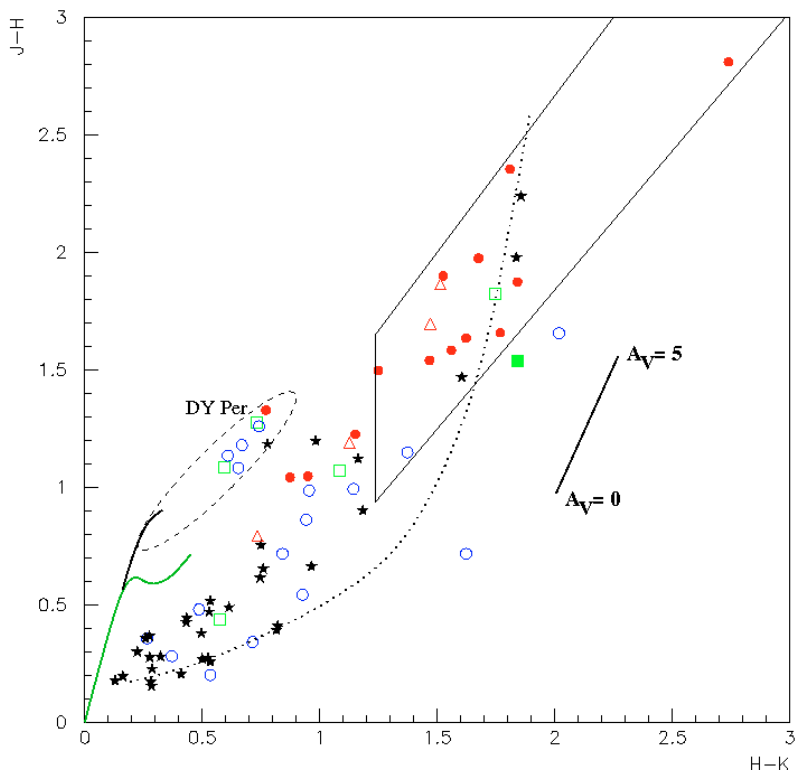

Fig. 5. The $J-H$ versus $H-K$ colour diagram. All the known RCB stars, including those of DY Per type, are represented. The black stars are the 31 confirmed Galactic RCBs listed by (Clayton 1996) plus ES Aql and V2552 Oph. The 4 open red triangles are the ones recently found by Zaniewski et al. (2005). The blue circles are the 17 RCB stars found in the LMC (Alcock et al. 2001). The green open squares are the 5 RCBs found in the SMC (Tisserand et al. 2004) and the full green square is the SMC RCB star MSX-SMC-014 (Kraemer et al. 2005). The 14 new EROS-2 Galactic RCB stars are indicated with red dots. The location of the DY Per stars is delimited by the dashed ellipse, which also encloses the area occupied by most carbon-rich stars. The dotted curve corresponds to the combination of blackbodies consisting of a $5500 \mathrm{~K}$ star and a $1000 \mathrm{~K}$ dust shell in various proportions ranging from all "star" to all "shell" (from Feast 1997). The area delimited by straight lines shows the zone in the 2MASS database from which stars in the EROS-2 fields were selected for light curve examination (see Sect. 4.3). The line on the right side represents the reddening vector from Rieke \& Lebofsky (1985). Also shown are the expected position (lines in the bottom-left side) for common dwarf (green) and giant (black) stars from Bessell \& Brett (1988).

than known RCB stars $\left(T_{\text {eff }} \sim 3800 \mathrm{~K}\right)$ and have unusually faint absolute magnitudes of $M_{V} \sim 0$. For three of them (EROS2-CGRCB-5, -9 and -14), we have an indication from the presence of a strong Ca II triplet in their spectrum (see Sect. 4.4.2) that they are actually hotter with a temperature more likely similar to classical RCBs $\left(T_{\text {eff }} \sim 5000 \mathrm{~K}\right.$, which corresponds to an intrinsic $V-I$ colour of $\sim 1.2$ ). Therefore these 3 RCB stars are certainly subject to a supplementary extinction. We retain 3 hypotheses. First, they could be located beyond the Galactic Bulge at a maximum distance of $\sim 14 \mathrm{kpc}^{7}$. We have thus assumed that the supplementary extinction comes from dust located beyond the Bulge. Second, these 3 RCBs may still be located inside the Bulge, but have circumstellar extinctions that are higher than those for known RCBs $\left(A_{V_{\text {circum }}} \sim 3 \mathrm{mag}\right.$ is needed). Thus, we would have not observed the maximum luminosity of these RCBs. The reddening vector due to a carbon shell is different to the interstellar one: a factor of 3.5 in the slope can be observed in Fig. 6. The reddening vectors due to carbon clouds have been estimated for three of the new RCB stars during a decline ${ }^{8}$. Third, there may be a higher foreground extinction due to compact interstellar dust clouds that happen to be in the line of sight ${ }^{9}$. However, we consider this possibility the least likely as the 3 Galactic RCBs would still be located in the colour magnitude diagram at about 1.3 mag below the LMC ones (if $T_{\text {eff }} \sim 5000 \mathrm{~K}$ ), implying that their location is behind the Bulge without extra reddening in the line of sight. We would thus be confronted with the improbable case where only the 3 RCB stars located beyond the Bulge in our new sample would be affected by a compact foreground cloud in their line of sight. We note also that if the last hypothesis is true, we should observe the converse, some RCB stars that have been over-corrected due to a lower extinction than average. But this is not the case.

In the case of EROS-CG-RCB-12, we have already mentioned in Sect. 4.4.2 that this star is intrinsically cooler than the other RCBs. Its position in Fig. 7 is therefore less surprising. It could be similar to the cool RCB star discovered in the SMC, EROS2-sm0067m28134b ${ }^{10}$ (Tisserand et al. 2004; Kraemer et al. 2005). However, the calculated absolute

\footnotetext{
7 After reddening correction due to the supplementary extinction, $A_{V_{\text {supp }}} \sim 2$ mag, the three RCBs are still located in the colour magnitude diagram (Fig. 7) at about 1.3 mag below the LMC ones.

8 We selected only three RCBs based on the colour variation during decline (see Figs. 9-9): we did not retain some RCBs that become bluer due to a blend with a bluer star.

9 This can be observed in one case: the chart of the star EROS2-CGRCB-5 (Fig. 10) shows a local high extinction area close to its position. 10 We note that the SMC star EROS2-sm0067m28134b may not have reached its maximum brightness in the EROS-2 light curve, as the maximum observed lasted only a few days. If so, this cool SMC RCB star should also be brighter and bluer.
} 

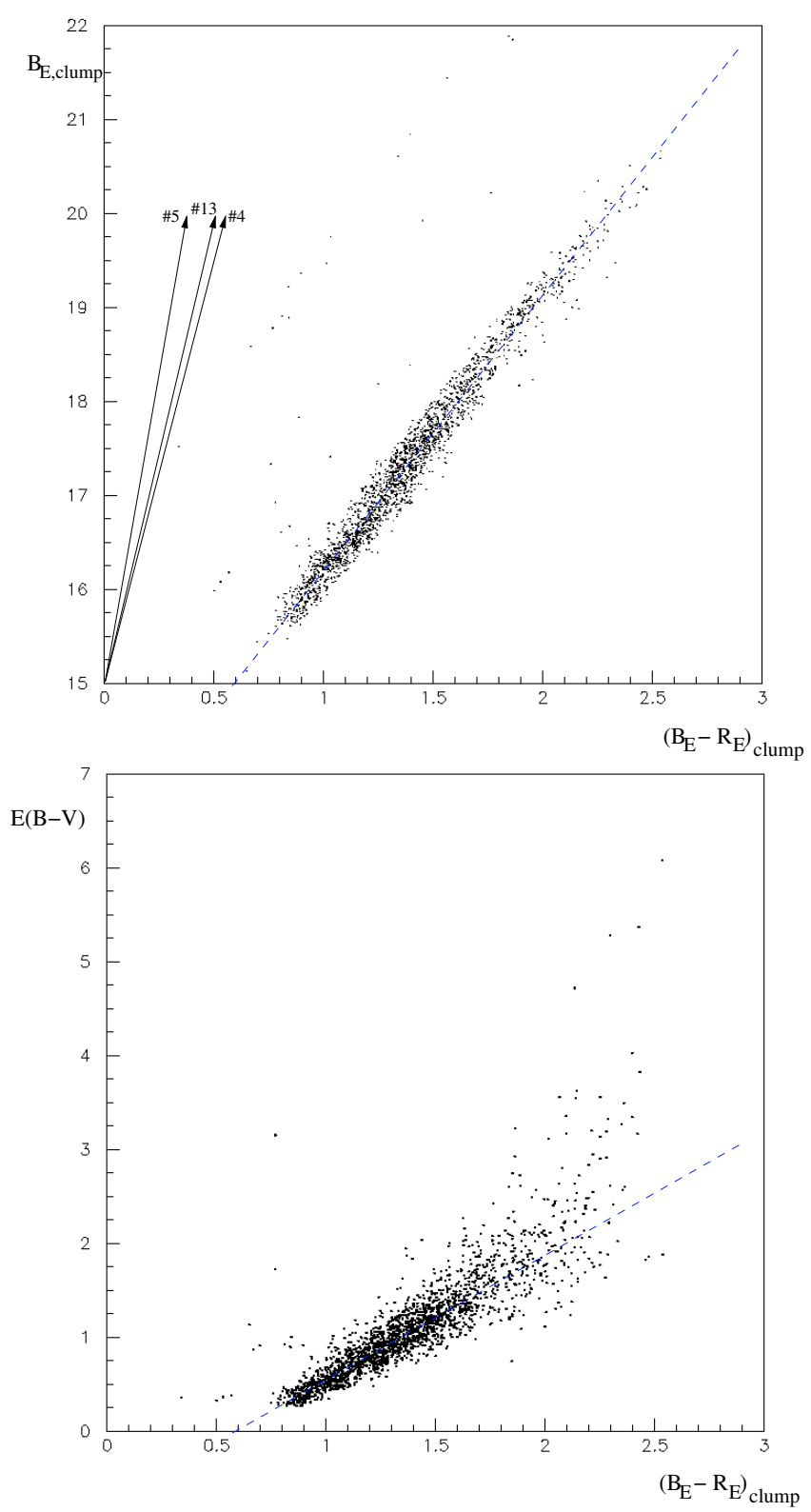

Fig. 6. Top panel: apparent magnitude $B_{\mathrm{E}, \mathrm{clump}}$ vs. $\left(B_{\mathrm{E}}-R_{\mathrm{E}}\right)_{\text {clump }}$ for the centre of the red giant clump in each quarter-CCD subfield. The expected linear relation due to reddening can be observed. The vectors on the left side represent the absorption due to carbon cloud, estimated for three RCBs during a decline. Bottom panel: the reddening $E(B-V)$ to the Bulge derived from the COBE/DIRBE data by Schlegel et al. (1998) versus $\left(B_{\mathrm{E}}-R_{\mathrm{E}}\right)_{\text {clump }}$.

magnitude with a Bulge distance hypothesis, $M_{V} \sim 0$, is unusual. As for the previous three RCB stars, it is more likely that EROS-CG-RCB-12 has an unusually thick circumstellar shell or is located behind the Bulge: with an estimated low temperature of $T_{\text {eff }} \sim 4400 \mathrm{~K}$ (which corresponds to an intrinsic $V-I$ colour of $\sim 1.9$ ), the maximum distance found is also of the order of $\sim 14 \mathrm{kpc}$.

After extinction correction, the Galactic DY Per-like RCB star EROS2-CG-RCB-2 has a $V-I$ colour that corresponds to that of the Magellanic DY Per-like RCB stars (note also that the prototype DY Per star is very cool, $T_{\text {eff }} \sim 3500$, see Keenan \& Barnbaum 1997). However, it is 1-2 mag fainter than the DY Per-like RCB stars found in the SMC and LMC. This discrepancy may be due to EROS2-CG-RCB-2 being more distant,

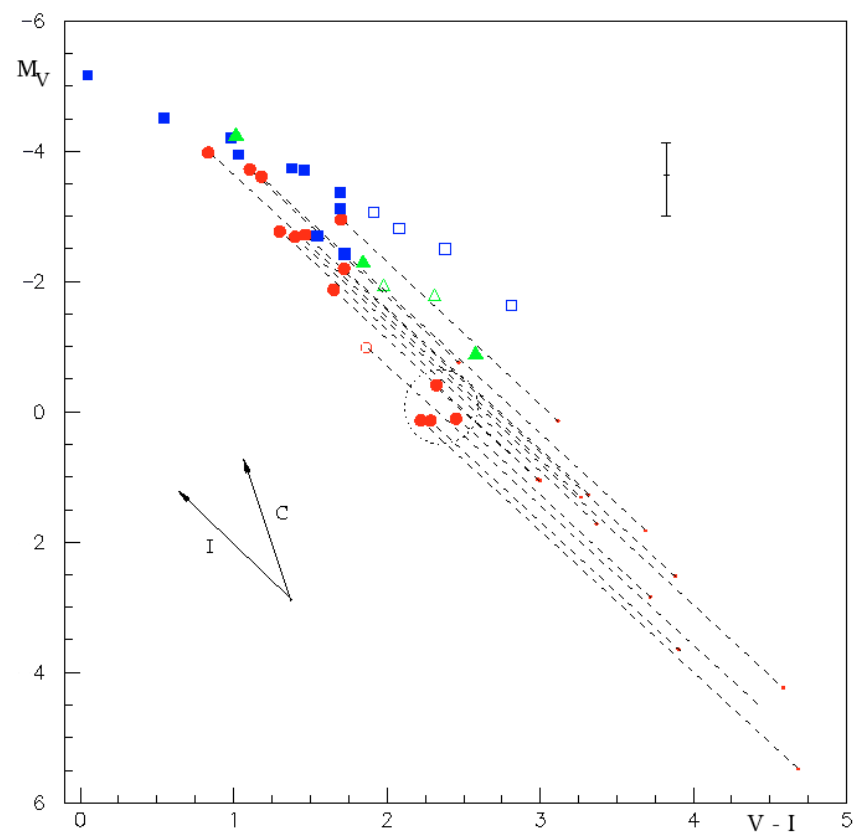

Fig. 7. Absolute magnitude at maximum, $M_{V}$, vs. $V-I$; full symbols represent common RCB stars, open symbols the DY Per-type RCB stars. The red circles are the newly discovered Galactic RCBs, assuming a galactocentric distance, after the interstellar reddening corrections shown by a dashed line for each star. The location of $10 \mathrm{LMC}$ RCBs, plus 4 DY Per-like RCBs, from Alcock et al. (2001) are indicated with blue squares. 3 SMC RCBs, plus 2 DY Per-like RCBs, from Tisserand et al. (2004) are shown by green triangles. All data are from the EROS-2 photometry. LMC and SMC RCB magnitudes have been corrected for their respective extinctions. The four Galactic RCBs surrounded by a dotted circle have an unusual position that can be explained by a thick circumstellar shell or a localisation behind the Bulge (see text). The vectors represent the reddening correction direction due to the interstellar medium (I) and a carbon shell (C) (see Fig. 6). The line in the up-right corner represents the lower and upper boundaries for $\mathrm{a} \pm 2 \mathrm{kpc}$ Galactic Bulge radius.

but correction for a (probable) additional extinction would make the star bluer and brighter.

Confirmation of the location of the stars could come from an equivalent width measurement of the interstellar lines of sodium (Na I, 5890.0, $5895.9 \AA$ ) and/or potassium (K I, $7699 \AA$ ) as there is a direct relation with reddening (see Munari \& Zwitter 1997). The KI line should be more helpful due to the high extinction $(E(B-V) \geq 0.4)$ and also because NaI D absorption lines are usually observed during declines in an RCB star spectrum (Clayton 1996). This study needs high resolution spectroscopy and has therefore not been performed with our data. We note that strong NaI D absorption lines are observed in our two highest resolution spectra of EROS2-CG-RCB-8 and EROS2-CG-RCB10 as well as in most of the low resolution spectra in the left panel of Fig. 4.

We now comment on two issues related to the empirical clump giant method used to correct our magnitudes for extinction. First, the calculated extinction is a regional average value, using clump giant stars located at less than 3 arcmin from each $\mathrm{RCB}$. If there is a localised low (high) extinction zone coinciding with the RCB, this method will over(under)-estimate the extinction. Second, most RCB stars have an intrinsic $V-I$ colour higher than that of the clump giant stars $\left((V-I)_{\text {Clump }}^{0} \sim 1.0\right)$. The applied absorption coefficient $R_{B_{\mathrm{E}}}=A_{B_{\mathrm{E}}} / E\left(B_{\mathrm{E}}-R_{\mathrm{E}}\right)$ should depend on the spectrum slope, which is measured by the intrinsic 
Table 7. Properties of the new Galactic RCB stars, including reddening estimates and derived absolute magnitudes and intrinsic colours.

\begin{tabular}{|c|c|c|c|c|c|c|c|c|c|c|c|}
\hline $\begin{array}{l}\text { RCB } \\
\text { Number }\end{array}$ & $\overline{R_{\mathrm{E}, \max }}$ & $\begin{array}{c}\left(\mathrm{d} R_{\mathrm{E}} / \mathrm{d} t\right)_{\max } \\
\mathrm{mag} \mathrm{day}^{-1}\end{array}$ & $\overline{\text { Drop } R_{\mathrm{E}}}$ & $\overline{B_{\mathrm{E}, \max }}$ & $\begin{array}{c}\left(\mathrm{d} B_{\mathrm{E}} / \mathrm{d} t\right)_{\max } \\
\text { mag day }^{-1}\end{array}$ & $\overline{\text { Drop } B_{\mathrm{E}}}$ & $E(B-V)^{a}$ & $\overline{A_{B_{\mathrm{E}}}}$ & $\Delta\left(B_{\mathrm{E}}-R_{\mathrm{E}}\right)$ & $\overline{M_{V}}$ & $\overline{(V-I)_{0}}$ \\
\hline 1 & 12.44 & 0.038 & $>8.3$ & 14.40 & 0.042 & $>7.1$ & 1.42 & 4.31 & 1.46 & -3.98 & 0.83 \\
\hline 2 & 14.48 & 0.021 & $>3.0$ & 17.10 & 0.019 & $>2.4$ & 2.56 & 4.43 & 1.50 & -0.98 & 1.87 \\
\hline 3 & 11.17 & 0.037 & $>6.9$ & 12.65 & 0.044 & 7.4 & 1.26 & 2.42 & 0.82 & -3.73 & 1.10 \\
\hline 4 & 12.54 & 0.067 & $>8.2$ & 14.75 & 0.052 & $>6.6$ & 2.03 & 4.43 & 1.50 & -3.61 & 1.18 \\
\hline 5 & 13.52 & 0.102 & 6.0 & 15.75 & 0.094 & $>5.7$ & 1.38 & 2.24 & 0.76 & 0.09 & 2.45 \\
\hline 6 & 12.75 & 0.034 & 6.2 & 14.77 & 0.036 & 6.0 & 2.40 & 3.66 & 1.24 & -2.77 & 1.30 \\
\hline 8 & 13.04 & nd & $>7.6$ & 15.37 & nd & $>6.0$ & 1.39 & 4.28 & 1.45 & -2.72 & 1.47 \\
\hline 9 & 15.20 & 0.033 & 2.6 & 18.01 & 0.035 & 1.8 & 3.14 & 4.37 & 1.48 & 0.13 & 2.22 \\
\hline 10 & 12.46 & 0.060 & 6.0 & 14.26 & 0.058 & 5.4 & 0.98 & 2.40 & 0.81 & -1.88 & 1.65 \\
\hline 11 & 12.35 & 0.058 & 5.8 & 14.34 & 0.060 & 5.1 & 1.14 & 2.83 & 0.96 & -2.20 & 1.72 \\
\hline 12 & 14.14 & 0.025 & 3.2 & 16.48 & 0.031 & 3.9 & 1.06 & 2.86 & 0.97 & 0.13 & 2.28 \\
\hline 13 & 11.42 & 0.043 & 5.1 & 13.29 & 0.066 & 6.2 & 1.38 & 2.51 & 0.85 & -2.94 & 1.70 \\
\hline 14 & 12.45 & 0.026 & $>8.3$ & 14.24 & 0.033 & $>7.2$ & 0.49 & 1.18 & 0.40 & -0.41 & 2.32 \\
\hline
\end{tabular}

nd $=$ not detected, ${ }^{a}$ from Schlegel et al. (1998).
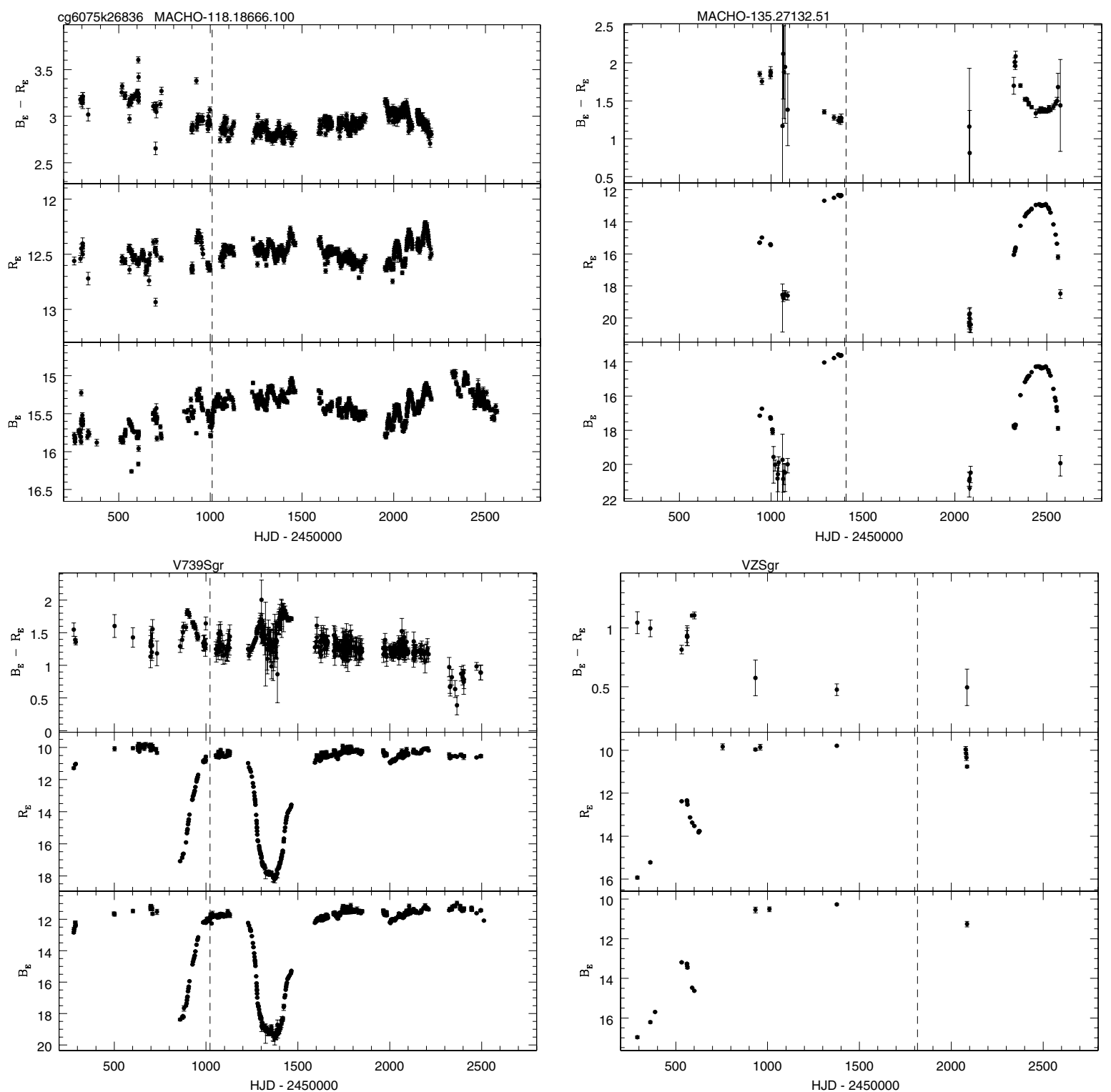

Fig. 8. EROS-2 light curves of MACHO-118.18666.100 (re-classified as an M giant star, see Sect. 3) and three known Galactic RCB stars, MACHO-135.27132.51, V739 Sgr and VZ Sgr. The dashed vertical lines indicate the 2MASS epochs. The photometry of the last three RCB stars has been reprocessed as these stars were either saturated or too faint on the original EROS-2 reference images. 

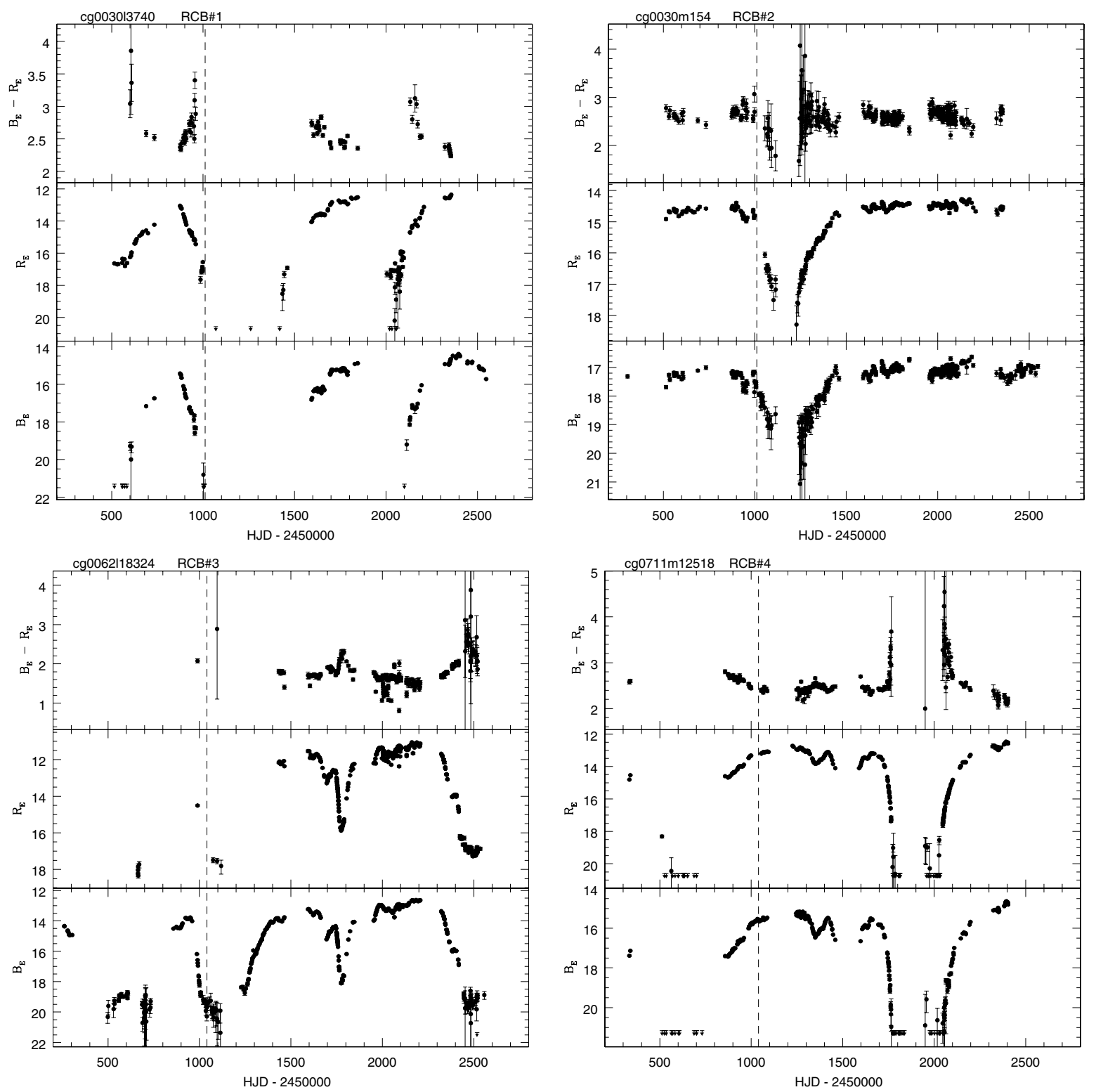

Fig. 9. Light curves of the 14 new RCB stars, one being a DY Per star (EROS2-RCB-CG-2). The arrows represent our detection limit. The dashed vertical lines indicate the $2 \mathrm{MASS}$ epochs. Top: $B_{\mathrm{E}}-R_{\mathrm{E}}$ colour vs. time; middle: $R_{\mathrm{E}}$ light curve; bottom: $B_{\mathrm{E}}$ light curve.

star colour. Hence, use of $R_{B_{\mathrm{E}}}$ derived from clump giants will not be exact for RCB stars. This effect may not be negligible, particularly with our wide EROS-2 bands (see Tammann et al. 2003 , for an example of the variation of $R$ for Cepheids). We note that the small difference in slope in Fig. 7, between the Magellanic and Galactic Bulge RCBs, may be due to this effect: the 3 Bulge RCB stars with $V-I \sim 1.0$ match well the position of the Magellanic RCBs in the colour magnitude diagram, whereas a small shift in luminosity is observed with the 6 redder Bulge RCBs, with $1.2<V-I<1.8$.

\subsection{Proper motion test}

We measured the proper motion for the 14 new RCBs using all available EROS-2 images, but no positive result was obtained. The study was complicated by blending which causes correlations between flux and position. This is particularly evident for the RCB star EROS2-CG-RCB-14, which is listed in the NOMAD catalogue with a significant proper motion $\left(\mu_{\alpha}=82 \pm 9\right.$ and $\mu_{\delta}=15.4 \pm 9$ mas/yr). We can explain this apparent movement by blending.

\subsection{Galactic latitude distribution}

We note that the density of RCB stars strongly increases towards the Galactic plane. We estimate a density in our fields of $\sim 0.6 \pm 0.2 \mathrm{RCB}$ per $\mathrm{deg}^{2}$ for low Galactic latitude $\left(1^{\circ}<|b|<3^{\circ}\right)$ and $\sim 0.09 \pm 0.05 \mathrm{RCB}$ per $\operatorname{deg}^{2}$ at higher latitude $\left(3^{\circ}<|b|<5^{\circ}\right)$. For this calculation, only the 14 new EROS-2 RCB stars were used and all fields higher than $|b|>5^{\circ}$ were not considered due to a dependence of our detection efficiency on Bulge RCBs absolute magnitude (see Sect. 4.2.1) ${ }^{11}$.

For the latitude range $|b|<5^{\circ}$, we applied the method of maximum likelihood to measure the scale height $h_{\mathrm{Bulge}}^{\mathrm{RCB}}$. We suppose a latitude distribution of the form $\exp (-b / h)$. If we use the

11 We note also that a larger fraction of less frequently observed fields are located at $|b|>5^{\circ}$ (see Fig. 3). 

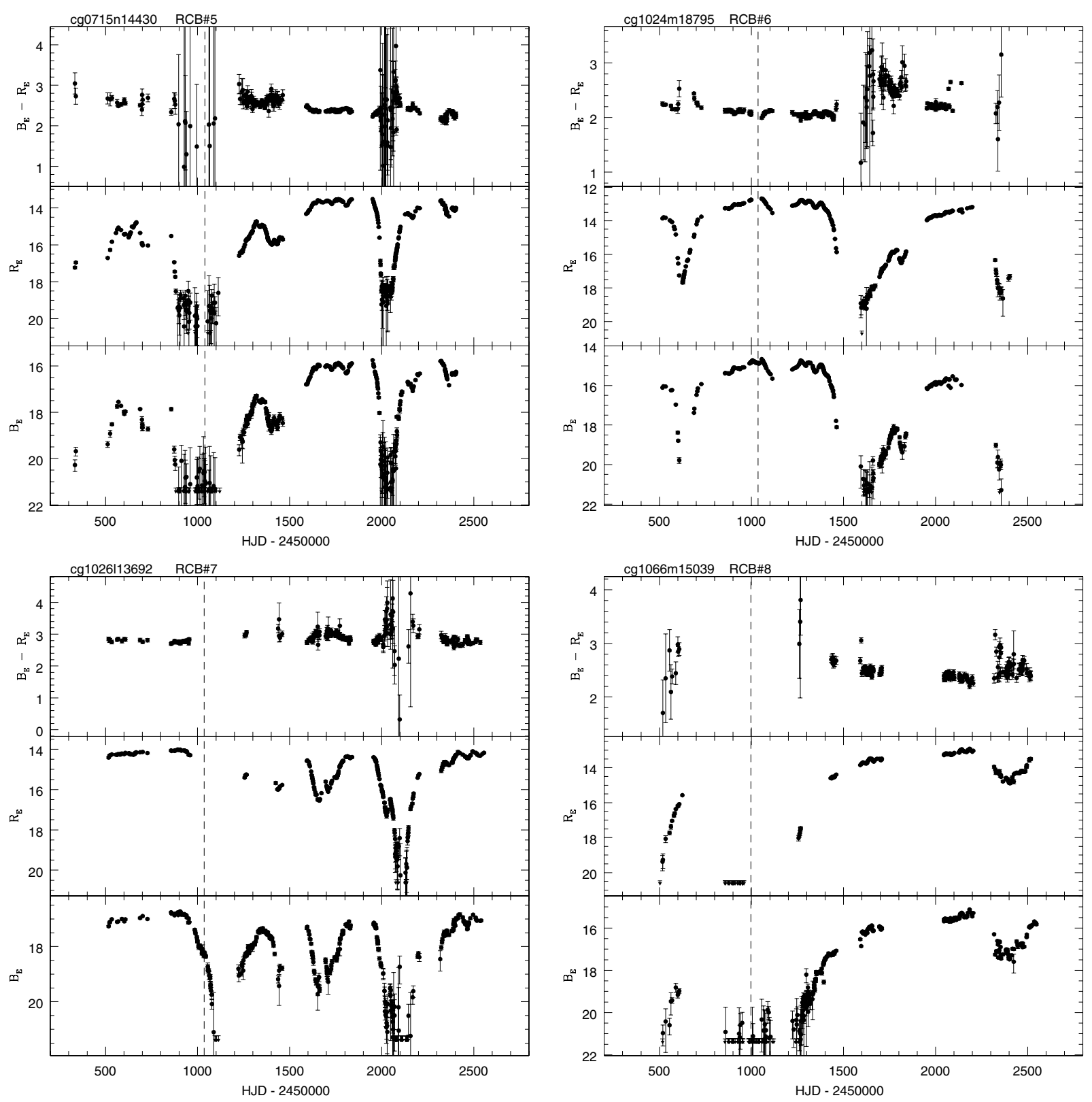

Fig. 9. continued.

14 new RCBs, we find $0.6<h_{\text {Bulge }}^{\mathrm{RCB}}<2.75 \mathrm{deg}(95 \%$ C.L.) with a most likely value of $1.0 \mathrm{deg}$ : It corresponds at the distance of the Galactic Centre to a scale height of $137_{-55}^{+239} \mathrm{pc}$. But if we restrict our analysis to the 9 new RCB stars that are most likely to be located inside the Bulge, we found smaller 95\% C.L. limits: $0.45<h_{\mathrm{Bulge}}^{\mathrm{RCB}}<2.0$ deg with a most likely value of $0.8 \mathrm{deg}$, i.e. $109_{-48}^{+137} \mathrm{pc}$ at the distance of the Galactic centre.

Such a small scale height is marginally consistent with the Bulge scale heights (270 to $360 \mathrm{pc}$ ) found by Dwek et al. (1995) from the COBE/DIRBE data, using three different exponential triaxial Bulge models. The measured scale height is significantly less than that expected for a thick disk ( $>1 \mathrm{kpc}$ ) and is similar to that for young/intermediate age thin-disk populations.

The small statistic prevent us from having a clear view of the distribution of the new RCBs; however, their distances and scale height seem to indicate a disk-like distribution inside the
Bulge. We note that such small scale heights have already been observed in the Galactic Bulge for OH/IR stars, which are oxygen-rich evolved stars (see Sevenster et al. 1995; Sevenster 1999). If confirmed, this result may help to constrain the age of the RCB stars, as a disk structure inside the Galactic Bulge could only be younger than the Galactic Bulge itself.

The observed scale height is lower than the one measured by Iben \& Tutukov (1985), 400 pc, using known RCB stars outside the Galactic Bulge and assuming $M_{\mathrm{Bol}}=-5$. This difference in scale height, between the inner part (Bulge) and the outer part (disk) of the Galaxy, has also been observed with $\mathrm{OH} / \mathrm{IR}$ stars (Sevenster 1999).

No RCB stars have been found in the 29 spiral arms fields. This is not a significant result, as few RCB stars would be expected in these fields. Assuming a disk scale length of $2.5 \mathrm{kpc}$ and the density found in our low Galactic latitude fields, we expected to find only $\sim 2$ RCB stars in the spiral arms fields. 

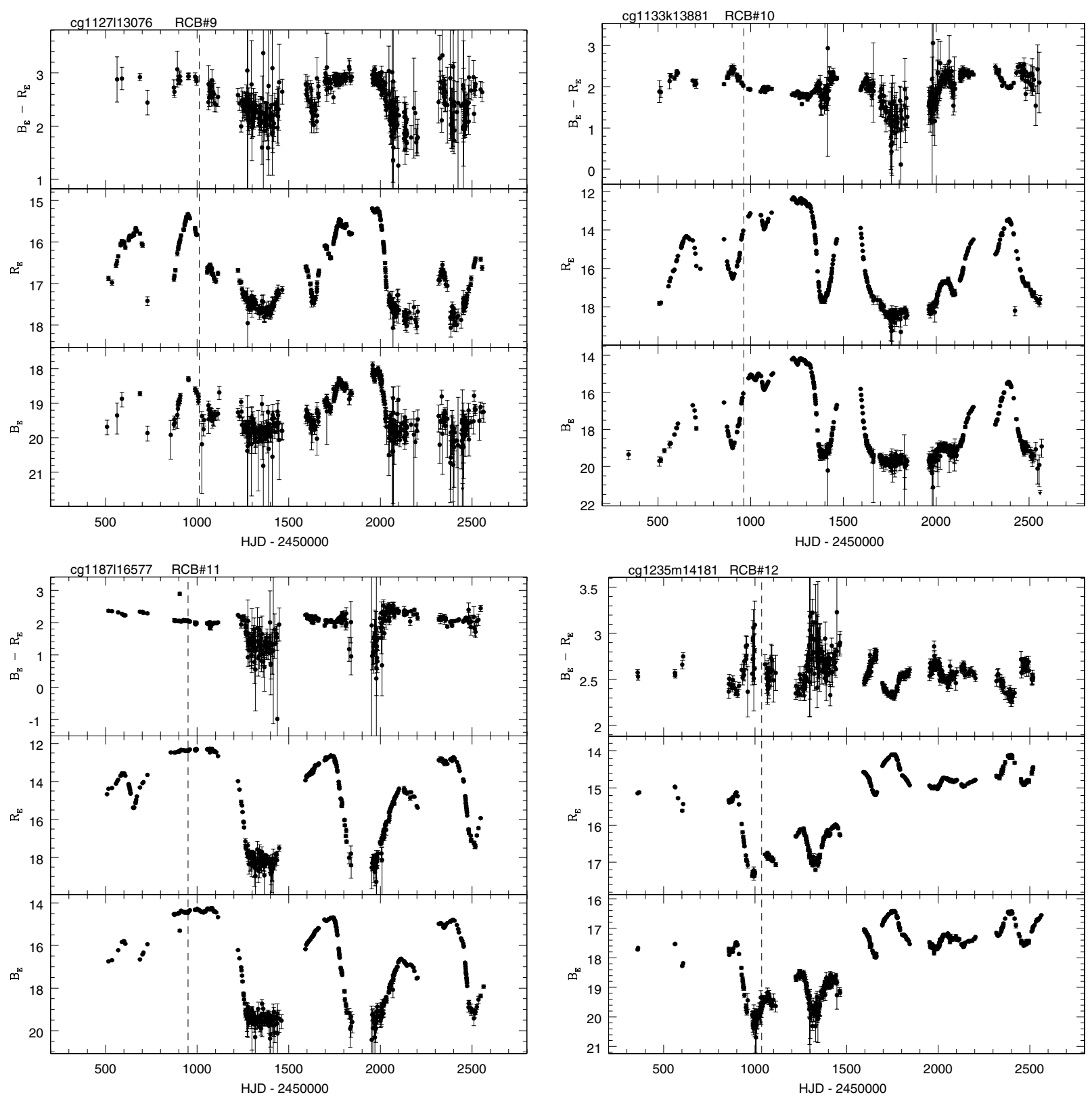

Fig. 9. continued.

\subsection{Individual stars}

EROS2-CG-RCB-9, EROS2-CG-RCB-10 and EROS2-CGRCB-11 all show multiple drops in brightness in their light curves, as well as carbon-rich spectra. They are undoubtedly members of the RCB class. None of these stars was previously catalogued as variable.

EROS2-CG-RCB-1 is the only new RCB star that has not been confirmed spectroscopically. Its spectrum is almost totally featureless, although some weak $\mathrm{CN}$ bands are present; the star was very faint during the observation on JD-2 454348. However its light curve has multiple declines of more than 8 mag, making it a strong RCB candidate. After extinction correction, EROS2CG-RCB-1 is the hottest RCB from the new sample. Its spectrum looks typical of a warm RCB star such as W Men (see Fig. 1 Alcock et al. 2001) with a fair amount of interstellar extinction $\left(A_{B_{\mathrm{E}}}=4.31\right)$.

EROS2-CG-RCB-2 is a DY Per star candidate due to its slow decline and symmetric recovery, plus a location in the $J-H$

versus $H-K$ diagram (Fig. 5) where all other DY Per stars are found. The spectrum confirms that the star is carbon-rich.

EROS2-CG-RCB-3 is the second hottest RCB star found in this analysis, after reddening correction. Its spectrum shows strong Ca II triplet lines as well as strong $C_{2}$ (Swan bands from 5500 to $6500 \AA$ ) and $\mathrm{CN}$ features. The lack of measurements in the red band before the year 2000 is due to an electrical problem in CCD number 2.

EROS2-CG-RCB-4, named Terz V 2046 by Terzan \& Ounnas (1988) was listed as a variable star due to its impressive luminosity change (close to 9 mag). Its R-band magnitude was measured at 9.2 in 1968, but fainter than 18 in 1981.

EROS2-CG-RCB-5 presents the quickest decline $\left(\sim 0.1\right.$ mag day $\left.^{-1}\right)$ with a fast recovery 200 days later and the highest IR excess with $J-H \sim 2.74$. Its spectrum shows strong Ca II triplet lines indicating that the star is hotter than the cool temperature estimated after a Bulge extinction correction. 

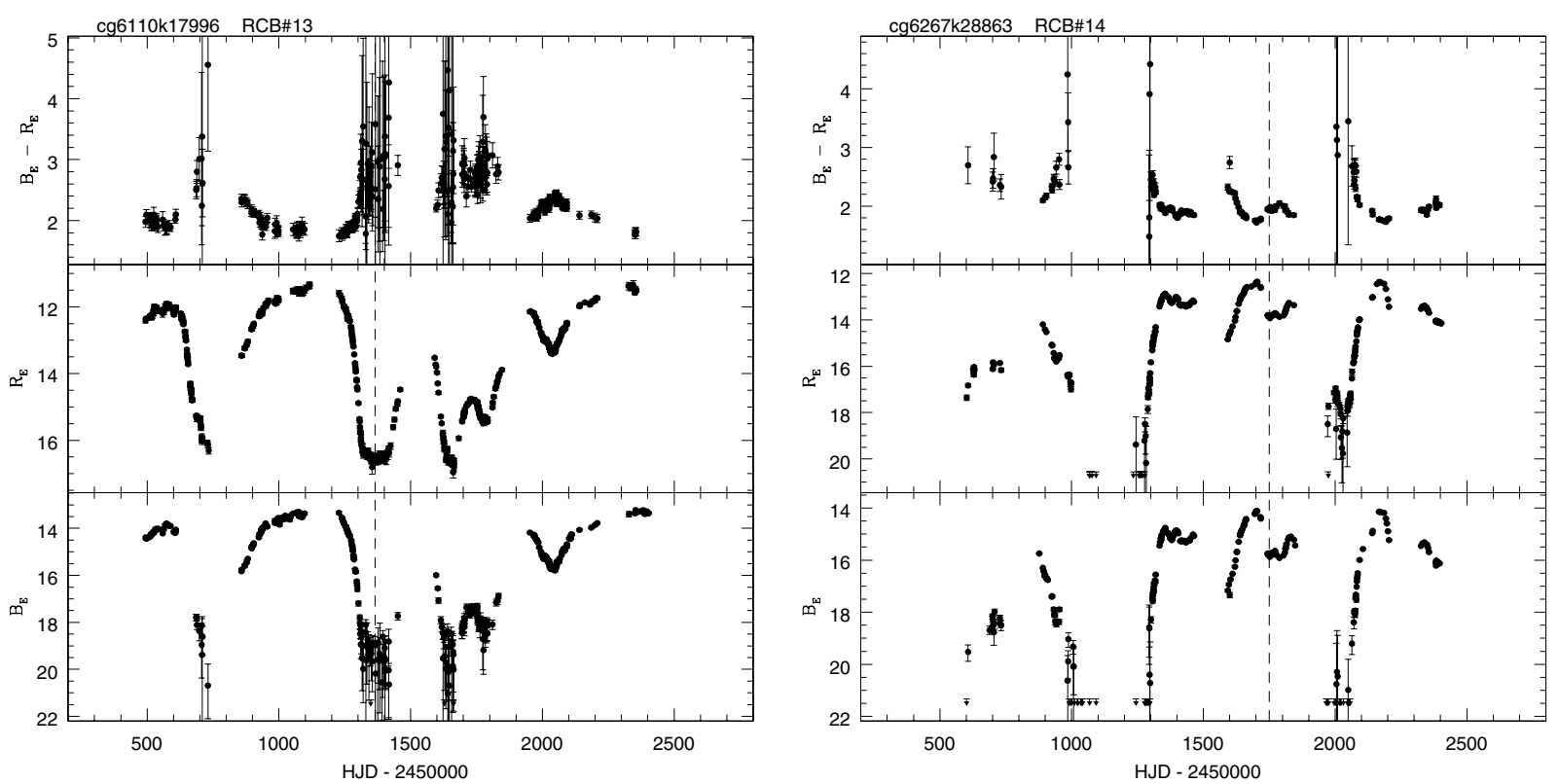

Fig. 9. continued.

EROS2-CG-RCB-6, named V1135 Sco in the GCVS ${ }^{12}$, was incorrectly classified as a Mira variable by Alard et al. (1996). No indication of periodicity is seen in its light curve.

EROS2-CG-RCB-7 was named Terz V 1680 by Terzan \& Ounnas (1988) with a variation in brightness of 5 mag between 1980 and 1976. Multiple aperiodic drops in luminosity (more than $6 \mathrm{mag}$ ) are visible in the EROS-2 light curve.

EROS2-CG-RCB-8 was named Terz V 2960 by Terzan \& Gosset (1991). Its brightness varied by more than 3.3 mag between 1968 and 1987. No sudden drop was observed in the EROS-2 light curve, but with a total amplitude change of more than $7.6 \mathrm{mag}$ in the $R_{\mathrm{E}}$ band, a slow recovery of its maximum brightness (nearly 2.5 years) and a carbon type spectrum, EROS2-CG-RCB-8 is a strong RCB star candidate.

EROS2-CG-RCB-12 shows one characteristic decline around JD-2 450900 along with many variations of large amplitude at maximum brightness. The total variation is slightly more than 3 mag over the 6.7 years of observations. Its spectrum shows clear evidence of carbon molecules in its atmosphere, making this star a strong RCB candidate. Surprisingly, its spectrum shows no Ca II triplet lines indicating that this RCB star is the coolest of the classical new RCB star found.

EROS2-CG-RCB-13 has been monitored by both EROS-2 and the MACHO experiment (MACHO-176.19607.1138). The first 2 drops observed by EROS-2 are also present in the MACHO light curve, while EROS-2 shows another drop around the epoch JD-2 451 100. Its spectrum indicates a carbon-rich atmosphere so it is also a strong RCB candidate.

EROS2-CG-RCB-14 has been catalogued in the NOMAD catalogue with a significant proper motion, which we ascribe to blending. This is the RCB star that shows the largest drop in luminosity in the new Galactic sample, with a variation of more than $8.3 \mathrm{mag}$ in the $R_{\mathrm{E}}$ band.

\section{Summary}

Our search for new RCB stars in the EROS-2 Galactic photometry resulted in the discovery of 14 new RCB stars, one being a

12 GCVS: General Catalogue of Variable Stars (Kukarkin et al. 1971).
DY Per star. The total number of confirmed Galactic RCB stars is therefore now 51. No RCB was found in the 29 fields monitored in the spiral arms. Overall, we estimate that our detection of RCB stars should be $\sim 60 \%$ complete for the majority of our survey.

MACHO-118.18666.100 has been misclassified as an RCB star. The new spectrum presented shows that it is an $\mathrm{M}$ giant star with light curve variations that resemble those of sequence-D variable stars.

If no interstellar reddening correction is applied, the new RCB sample would consist of the coolest RCB stars ever found. An extinction correction that assumes that the stars are located in the Bulge give intrinsic magnitudes and colours for 9 of the new RCBs that overlap those of the LMC and SMC. This indicates that they are indeed in the Galactic Bulge - the first RCB stars identified in the Bulge.

Concerning the remaining classical RCBs, 4 have the lowest colour temperatures and faintest luminosities (EROS2-CGRCB-5, -9, -12 and -14), assuming that the stars are located in the Bulge. However, these unusual properties may be explained either by an unusual thick circumstellar carbon shell or by a location beyond the Bulge at a maximum distance of $\sim 14 \mathrm{kpc}$. The supplementary distance would thus give rise to an extra reddening. From the study of the Ca II triplet strength, we suggest that EROS2-CG-RCB-5, -9, and -14 have typical RCB temperatures $\left(T_{\text {eff }} \sim 5000 \mathrm{~K}\right)$ and that EROS2-CG-RCB-12 is the coolest of the classical RCBs found.

Finally, we note that the density of RCB stars seems to increase strongly as the galactic plane is approached. We measured a small scale height of $109_{-48}^{+137} \mathrm{pc}$ at the distance of the Galactic Centre using the 9 RCBs that are more likely to be located inside the Bulge. This small value is not consistent with measured Bulge scale height values and suggests a disk-like distribution inside the Bulge. Our derived distribution suggests that the RCB star density should be higher than one per $\operatorname{deg}^{2}$ for $|b|<2^{\circ}$ towards the Galactic Bulge. Hence, we suggest that the next generation of infrared variable star surveys of the Bulge 


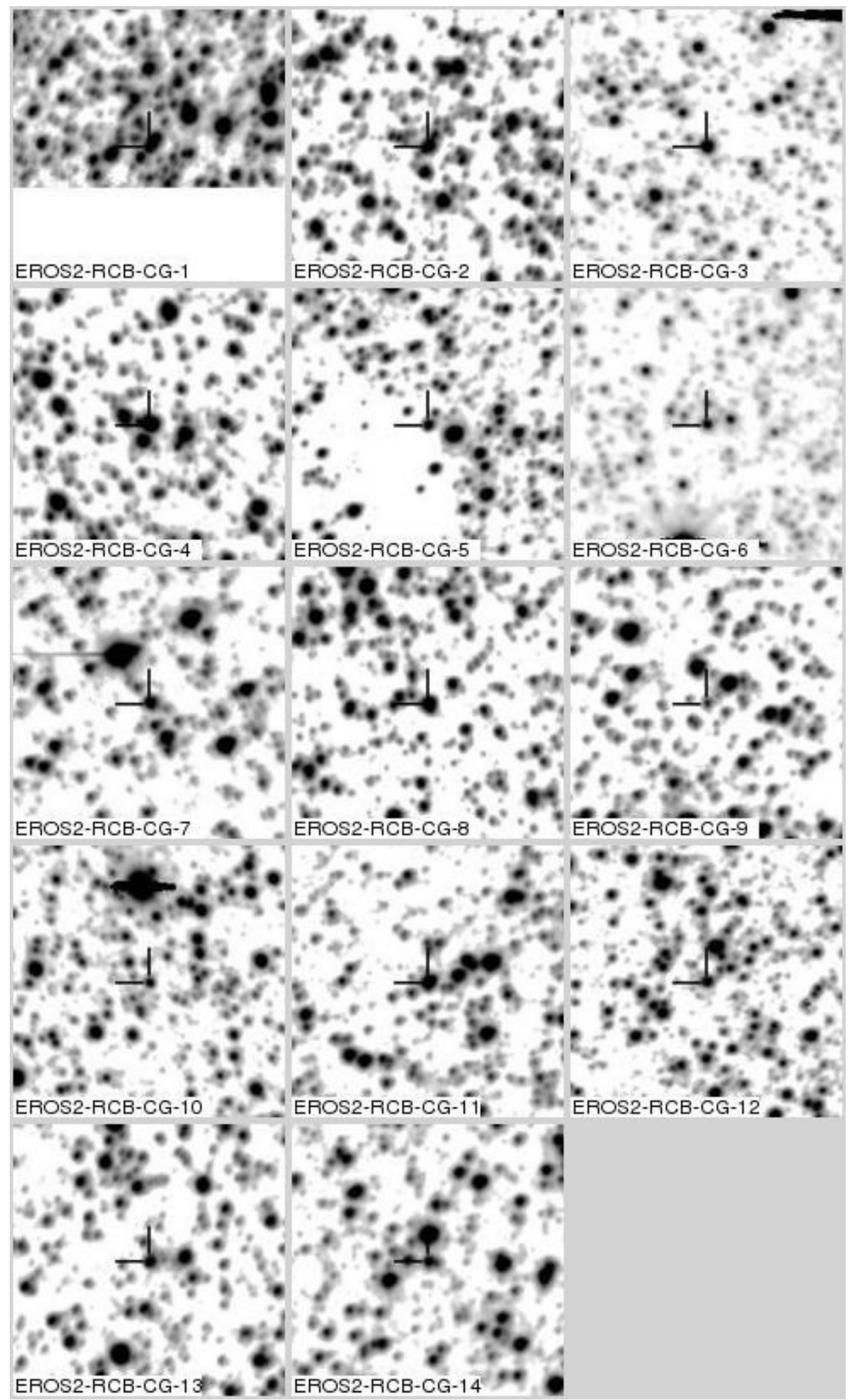

Fig. 10. Charts of the new Galactic RCB stars $\left(2^{\prime} \times 2^{\prime}\right)$. North is up, East is to the left. 
(e.g. VISTA ${ }^{13}$ ) should be able to discover many RCB stars near the Galactic Centre.

Acknowledgements. This paper is dedicated to the memory of Alain Milsztajn (1955-2007).

We would like to thank the referee, G. Clayton, for his comments that improved the paper and K.C. Freeman for useful discussions. This publication makes use of data products from the Two Micron All Sky Survey, which is a joint project of the University of Massachusetts and the Infrared Processing and Analysis Centre California Institute of Technology, funded by the National Aeronautics and Space Administration and the National Science Foundation. The Denis data have also been used. DENIS is the result of a joint effort involving human and financial contributions of several Institutes mostly located in Europe. It has been supported financially mainly by the French Institut National des Sciences de l'Univers, CNRS, and French Education Ministry, the European Southern Observatory, the State of Baden-Wuerttemberg, and the European Commission under networks of the SCIENCE and Human Capital and Mobility programs, the Landessternwarte, Heidelberg and Institut d'Astrophysique de Paris. J.A. acknowledges support from the Danish Natural Science Research Council.

\section{References}

Alard, C., Terzan, A., \& Guibert, J. 1996, A\&AS, 120, 275

Alcock, C., Allsman, R. A., Alves, D. R., et al. 2001, ApJ, 554, 298

Alksnis, A. 1994, Balt. Astron., 3, 410

Alksnis, A., Balklavs, A., Dzervitis, U., et al. 2001, Balt. Astron., 10, 1

Ansari, R. 1996, Vist. Astron., 40, 519

Arce, H. G., \& Goodman, A. A. 1999, ApJ, 512, L135

Asplund, M., Lambert, D. L., Kipper, T., Pollacco, D., \& Shetrone, M. D. 1999, A\&A, 343, 507

Azzopardi, M., Rebeirot, E., Lequeux, J., \& Westerlund, B. E. 1991, A\&AS, 88, 265

Belczynski, K., Bulik, T., \& Ruiter, A. J. 2005, ApJ, 629, 915

Bessell, M. S., \& Brett, J. M. 1988, PASP, 100, 1134

Clayton, G. C. 1996, PASP, 108, 225

Clayton, G. C., \& De Marco, O. 1997, AJ, 114, 2679

Clayton, G. C., Geballe, T. R., Herwig, F., Fryer, C., \& Asplund, M. 2007, ApJ, 662,1220

Clayton, G. C., Hammond, D., Lawless, J., et al. 2002, PASP, 114, 846

Clayton, G. C., Kerber, F., Pirzkal, N., et al. 2006, ApJ, 646, L69

Cottrell, P. L., \& Lawson, W. A. 1998, Publ. Astron. Soc. Austr., 15, 179

de Laverny, P., \& Mékarnia, D. 2004, A\&A, 428, L13

Derue, F., Afonso, C., Alard, C., et al. 2001, A\&A, 373, 126

Dutra, C. M., Santiago, B. X., Bica, E. L. D., \& Barbuy, B. 2003, MNRAS, 338, 253

Dwek, E., Arendt, R. G., Hauser, M. G., et al. 1995, ApJ, 445, 716

Egan, M. P., Price, S. D., Kraemer, K. E., et al. 2003, VizieR Online Data Catalog, 5114, 0

Eisenhauer, F., Genzel, R., Alexander, T., et al. 2005, ApJ, 628, 246

Feast, M. W. 1997, MNRAS, 285, 339

Gonzalez, G., Lambert, D. L., Wallerstein, G., et al. 1998, ApJS, 114, 133
Hamadache, C., Le Guillou, L., Tisserand, P., et al. 2006, A\&A, 454, 185 Hesselbach, E., Clayton, G. C., \& Smith, P. S. 2003, PASP, 115, 1301 Iben, Jr., I., \& Tutukov, A. V. 1985, ApJS, 58, 661

Iben, I. J., Tutukov, A. V., \& Yungelson, L. R. 1996, ApJ, 456, 750

Jurcsik, J. 1996, Acta Astron., 46, 325

Keenan, P. C., \& Barnbaum, C. 1997, PASP, 109, 969

Kilkenny, D. 1997, The Observatory, 117, 205

Kraemer, K. E., Sloan, G. C., Wood, P. R., Price, S. D., \& Egan, M. P. 2005, ApJ, 631, L147

Kukarkin, B. V., Kholopov, P. N., Pskovsky, Y. P., et al. 1971, in General Catalogue of Variable Stars, 3rd edn.

Landolt, A. U. 1992, AJ, 104, 372

Lawson, W. A., \& Cottrell, P. L. 1990, in Confrontation Between Stellar Pulsation and Evolution, ed. C. Cacciari, \& G. Clementini, ASP Conf. Ser., 11,566

Lawson, W. A., Cottrell, P. L., Kilmartin, P. M., \& Gilmore, A. C. 1990, MNRAS, 247, 91

Leão, I. C., de Laverny, P., Chesneau, O., Mékarnia, D., \& de Medeiros, J. R. 2007, A\&A, 466, L1

Lloyd Evans, T. 1991, MNRAS, 249, 409

Lloyd Evans, T., Kilkenny, D., \& van Wyk, F. 1991, The Observatory, 111, 244 Milone, L. A. 1990, Ap\&SS, 172, 263

Munari, U., \& Zwitter, T. 1997, A\&A, 318, 269

Paczynski, B. 1986, ApJ, 304, 1

Pojmanski, G. 1997, Acta Astron., 47, 467

Pollard, K. R., Cottrell, P. L., \& Lawson, W. A. 1994, MNRAS, 268, 544

Rahal, Y. R. 2003, Ph.D. Thesis, Université Paris 6

Renzini, A. 1990, in Confrontation Between Stellar Pulsation and Evolution, ed.

C. Cacciari, \& G. Clementini, ASP Conf. Ser., 11, 549

Richer, H. B. 1971, ApJ, 167, 521

Rieke, G. H., \& Lebofsky, M. J. 1985, ApJ, 288, 618

Rodgers, A. W., Conroy, P., \& Bloxham, G. 1988, PASP, 100, 626

Schlegel, D. J., Finkbeiner, D. P., \& Davis, M. 1998, ApJ, 500, 525

Sevenster, M. N. 1999, MNRAS, 310, 629

Sevenster, M. N., Dejonghe, H., \& Habing, H. J. 1995, A\&A, 299, 689

Skrutskie, M. F., Cutri, R. M., Stiening, R., et al. 2006, AJ, 131, 1163

Stanek, K. Z. 1998, ArXiv Astrophysics e-prints

Stephenson, C. B. 1989, Publ. of the Warner \& Swasey Observatory, 3, 53

Sumi, T. 2004, MNRAS, 349, 193

Tammann, G. A., Sandage, A., \& Reindl, B. 2003, A\&A, 404, 423

Terzan, A., \& Gosset, E. 1991, A\&AS, 90, 451

Terzan, A., \& Ounnas, C. 1988, A\&AS, 76, 205

Tisserand, P., Le Guillou, L., \& the EROS-2 Coll. 2007, A\&A, 469, 387

Tisserand, P., Marquette, J. B., Beaulieu, J. P., et al. 2004, A\&A, 424, 245

Trimble, V., \& Kundu, A. 1997, PASP, 109, 1089

Udalski, A., Szymanski, M., Kubiak, M., et al. 2002, Acta Astron., 52, 217

Webbink, R. F. 1984, ApJ, 277, 355

Wood, P. R., Alcock, C., Allsman, R. A., et al. 1999, in Asymptotic Giant Branch

Stars, ed. T. Le Bertre, A. Lebre, \& C. Waelkens, IAU Symp., 191, 151

Zaniewski, A., Clayton, G. C., Welch, D. L., et al. 2005, AJ, 130, 2293

Zaritsky, D., Harris, J., Thompson, I. B., Grebel, E. K., \& Massey, P. 2002, AJ, 123,855

\footnotetext{
13 Visible and Infrared Survey Telescope for Astronomy, URL: http: //www.vista.ac.uk/
} 